\title{
LE TRAIETTORIE RETICOLARI DELL'INNOVAZIONE TERRITORIALE
}

\author{
MaUro Lombardi e Nicolò BellancA*
}

\section{The reticular paths of territorial innovation}

\begin{abstract}
Traditions of studies dating back to Marshall, Porter and Krugman interpreted Local socioeconomic systems (LSS) mainly considering the spatial proximity of the actors. It was the local anchorage that enabled an increase in specific forms of external economies, competitive advantages and endogenous dynamics. Therefore, in the last decades, these local systems have gone through multiple changes in a multi-dimensional scale. The new structural connotations - including cognitive proximity, the task-based competition, the complementarity of formal contracts and informal agreements in business partnerships, trans-local networks require a different theoretical framework and involve different policy implications.

The theoretical framework focuses on co-evolution of technologies, organizational models, cultures and institutions. Among the multiple trajectories made possible by the co-evolution, each SSL is both related to a socio-technical system that restricts its dynamics of change, and plugged into paths along which can access in a discontinuous way to far techno-economic horizons.

Therefore the policy implications must refer to innovative trajectories that the existing global socio-technical transition makes possible for a specific group of SSL, which is Tuscany in our case. At the strategic and operative level - considering political and social limits of the society we are considering - we try to catch some "bottlenecks" which obstruct the perception and the pursuit of long-term collective interests. These blocks relate to myopia, both of entrepreneurs and of public institutions, in respect of the scientific-technical potential and effectively evolutionary paths that would be convenient to take; the inadequacy of institutional forms within which commons are produced and managed; the lack of appropriate ways to capitalize innovative enterprises. For each of these lock-in we make constructive and viable proposals.
\end{abstract}

Keywords: District, Local socio-economic system, Socio-technical transition, Lock-in, Tuscany.

JEL Classification: B52; L25; L53; O33.

\footnotetext{
- Dipartimento di Scienze Economiche, Università di Firenze. mauro.lombardi@unifi.it e nicolo.bellanca@unifi.it Questo paper è la versione draft del keynote speech per il Convegno dell'Associazione Italiana di Sociologia, Sezione Economia, Lavoro e Organizzazione, che si è svolta a Firenze tra I'8 e il 10 luglio 2010. Ringraziamo Filippo Barbera e il Direttivo dell'AIS-ELO per l'invito e tutti coloro che sono intervenuti con osservazioni, critiche e commenti.
} 
"Colui che non si preoccupa delle cose future è destinato ad affliggersi per quelle presenti"

\section{PRemessa}

La centralità dei sistemi socio-economici locali (d'ora in avanti: SSL) nell'odierna dinamica della globalizzazione è quasi unanimemente riconosciuta ${ }^{1}$. I SSL sono variamente definiti da numerosi approcci teorici: distretti industriali, clusters, milieux innovativi, sistemi d'innovazione nazionali/regionali/locali, città creative, ed altri ancora. Nella Prima parte del saggio tratteremo alcuni dei mutamenti da essi attraversati negli ultimi decenni, delineando le nuove peculiarità strutturali che riteniamo oggi indispensabili alla loro teorizzazione. Argomenteremo inoltre che, essendo l'evoluzione dei SSL strettamente connessa a dinamiche multi-dimensionali che agiscono a molteplice scala, alcuni importanti schemi interpretativi del passato, centrati sulla prossimità territoriale degli attori, appaiono ormai un ostacolo alla comprensione se non viene riformulato lo stesso concetto di prossimità. Questa tesi critica sarà declinata propositivamente nella Seconda parte, dove cercheremo di delineare la pluralità morfologica e la molteplicità delle regole di funzionamento dei SSL. Nella Terza parte verrà effettuato un tentativo di tratteggiare lo spazio dei maggiori trend economici prevedibili, indicando come ciascun SSL possa esplorare in tale spazio le proprie potenzialità d'innovazione. Infine, nella Quarta parte trarremo alcune implicazioni operative e avanzeremo dei suggerimenti di policy che possano valere hic et nunc, ossia nelle effettive difficili condizioni attuali di un SSL italiano (prenderemo quale esempio la Toscana).

\section{Prima parte. Traiettorie Reticolari e trasformazioni teCNO-ECONOMICHe}

\subsection{Alcune coordinate di fondo: macro-trend emersi negli ultimi decenni}

In questo paragrafo saranno anzitutto descritti cinque macro-trend emersi negli ultimi decenni a livello globale: 1) centralità assunta dalla "tecnologia intellettuale di produzione", 2) conseguente creazione di un global intelligent space, 3) sviluppo di global value chains nella produzione di beni e global networks in campo tecnico-

\footnotetext{
1 I SSL possono coincidere con Paesi, Regioni o altre unità territoriali definite a scala ritenuta
} economicamente significativa. Nell'ultima Parte di questo paper faremo riferimento empirico alla Toscana. 
scientifico, che tende a caratterizzarsi come spazio combinatorio di flussi informativi e di intersezioni dinamiche tra spazi e sotto-spazi di ricerca, 4) processo di continuo avanzamento della frontiera tecnico-scientifica, 5) spinte endogene all'autoorganizzazione dei processi tecnico-scientifici.

Saranno quindi illustrati gli effetti delle traiettorie indicate a tre livelli di analisi e descrizione: micro, meso e macro-scopici. Il primo attiene alla trasformazione dei cicli e delle fasi di produzione, il secondo concerne cambiamenti nelle proprietà sistemiche dei tradizionali SSL, mentre il terzo riguarda la dinamica di assetti socio-economici pervasi di sistemi socio-tecnici e di processi multi-livello e multi-scalari.

Nella prospettiva indicata il punto di partenza della riflessione non può che essere l'analisi dei cambiamenti del panorama tecno-economico nella seconda metà del secolo scorso, con l'intento di mettere in evidenza le coordinate fondamentali di una dinamica che influenza profondamente i processi evolutivi dei sistemi territoriali. L'esame di una rilevante letteratura consente di estrarre cinque macro-trend, emersi nel periodo più recente, il cui esplicarsi ha indotto profonde trasformazioni strutturali nelle economie di tutto il mondo, anche se con ritmi e intensità variabili. Le traiettorie che stiamo per descrivere in effetti corrispondono a vere e proprie discontinuità, innescate da punti di svolta conseguenti all'accumulo di fattori tali da ingenerare cambiamenti sistemici. Vale quindi la pena partire dalla descrizione di quelle che possiamo definire vere e proprie nuove coordinate generali dei processi di mutamento, con un chiarimento preliminare: ci soffermeremo su fenomeni e processi aventi caratteri di irreversibilità. Di essi cercheremo di delineare le conseguenze sul piano teorico ed empirico.

La prima traiettoria va colta nel punto di accumulazione di una serie di sviluppi tecnico-scientifici, ovvero nell'emergere della "tecnologia intellettuale di produzione" (Bell, 1999: 27-33). Questa espressione denota un passaggio decisivo nella storia degli esseri viventi: la conoscenza teorica viene codificata, per poi essere trasformata in un algoritmo incorporato in una macchina, che per questa via si trasforma da dispositivo puramente meccanico in strumentazione in grado di elaborare informazione. L'esistenza di tali meccanismi e la nascita delle tecnologie dell'informazione presentano immediatamente alcune proprietà decisive. Innanzitutto penetrano in ogni sfera della vita economica e sociale, dove generano mutamenti grazie alla loro «abilità di combinarsi con altre tecnologie, sfruttando complementarità con effetti a cascata. Essi sono diventati così pervasivi da meritarsi la definizione di General Purposive Technologies »(Mokyr, 2002: 41). Emerge poi la capacità di 
acquisire, trasmettere e modificare volumi crescenti di informazione, i quali sono ampliati dalle interazioni tra i possessori, al tempo stesso potenzialmente recettori e produttori di informazione. Questa capacità è intrinsecamente connessa ad un dato di fondo: la conoscenza teorica trasformata in algoritmo incorporato in una macchina non rende la stessa conoscenza un oggetto statico, in quanto l'algoritmo è riprogrammabile e -anche se non modificato- interagisce con la conoscenza continuamente elaborata dagli agenti (economici e non) innescando un processo di crescita esponenziale. Riflessioni originali di Bell in materia sono state riprese e sviluppate da Castells (2002: 32): «La peculiarità della rivoluzione tecnologica attuale consiste non nella centralità della conoscenza e dell'informazione, ma nell'applicazione della conoscenza e dell'informazione a dispositivi per la generazione della conoscenza e per l'elaborazione/comunicazione dell'informazione, in un ciclo di feedback cumulativo tra innovazione e usi dell'innovazione». Il nucleo propulsivo di questa «valanga tecnologica mai scatenatasi» (Freeman e Soete, 1994: 44) risiede dunque nella tecnologia intellettuale di produzione, la cui spinta dinamica deriva dall'innesco di processi conoscitivi iterati e dallo sviluppo di interazioni potenzialmente illimitate tra agenti. Questi ultimi sono infatti potenzialmente in grado di accedere a domini conoscitivi elaborati da altri grazie alla disponibilità di una crescente potenza computazionale. Proprio in tale aspetto risiede una delle novità più rilevanti ai nostri fini, perché lo sviluppo della tecnologia intellettuale di produzione modifica le strutture interattive in cui sono immersi individui, organizzazioni e Paesi.

Veniamo così alla seconda traiettoria, indotta dagli elementi appena indicati: ciò che la computer scientist Kathleen Carley (2000) ha definito global intelligent space. Quest'espressione sintetizza quattro caratteristiche precipue dell'epoca odierna: 1) Accesso ubiquitario all'informazione, ovunque e in qualunque momento essa sia prodotta, da parte di agenti (umani o artificiali), permettendo così a chiunque di poter modificare continuamente le basi cognitive delle proprie strategie di comportamento. 2) Flussi informativi crescenti, per tale via prodotti, accumulati e continuamente rielaborati in qualsiasi parte del mondo. 3) Intelligenza distribuita, intesa come elaborazione diffusa delle informazioni, dal momento che l'accesso ubiquitario a flussi informativi globali consente a varie tipologie di agenti lo sviluppo di capacità e competenze, differenziate a seconda dei contesti e delle strutture connettive multiscala in cui si inseriscono. 4) Pervasività di dispositivi in grado di elaborare informazione, in misura tale che le barriere tra mondo analogico e mondo digitale sono sempre meno impermeabili, dando così origine ad uno spazio intelligente. Al suo 
interno l'informazione digitalizzata favorisce il superamento di barriere fisiche attraverso l'emergere di una molteplicità di configurazioni socio-economiche, ovvero di sistemi che si sviluppano grazie a input multi-dimensionali e multi-scalari.

Su queste basi si afferma un terzo macro-trend: la creazione di network internazionali, che assumono tipologie e forme evolutive estremamente differenziate. Un numero rilevante di studi ha messo in luce i processi che hanno portato all'affermarsi in molti settori ${ }^{2}$ di global value chains e global production networks (GPN), che hanno inserito, nei cicli di produzioni di beni e servizi, aree e Paesi fino a pochi anni or sono sostanzialmente fuori dalla dinamica economica internazionale. Molte analisi si soffermano su una precisa valenza delle reti produttive globali, cioè la trasformazione di processi produttivi in processi tecno-economici che si sviluppano simultaneamente su più aree e contesti territoriali (divengono distribuiti), grazie a flussi informativi e feedback a scala crescente, favorendo così l'upgrading tecnologico di agglomerazioni economico-territoriali. Non sono peraltro mancate analisi critiche, incentrate sul comportamento talvolta "predatorio" dei GPN, che in realtà hanno attinto a capabilities sviluppate in ambiti locali, spesso alla ricerca unicamente di economie di costo più che a sostenere il loro avanzamento tecnico-produttivo (Bellandi, 2001).

L'evidenza empirica in proposito non è univoca, ma ciò è molto probabilmente dovuto proprio alla differenza di comportamento degli agenti che hanno creato i GPN, in quanto a volte orientati a strategie di "accaparramento di risorse locali" ed altre a disegni di sviluppo dei vantaggi competitivi derivanti non solo da economie di costo, bensì anche al perseguimento congiunto di competenze dinamiche e contenute remunerazioni degli input. Si pensi agli investimenti internazionali nell'industria hightech e nelle tecnologie dell'informazione in India; essi sono stati indubbiamente innescati dalla ricerca di elevati livelli di competenza, uniti a convenienze di costo. Nel caso della Cina hanno all'inizio agito soprattutto motivazioni inerenti ai costi, ma successivamente sembrano emergere anche elementi relativi all'impiego simultaneo di capabilities di crescente qualità.

Ai nostri fini è però opportuno sottolineare la tendenza sempre più marcata verso la creazione di network globali nel mondo tecnico-scientifico, che ha visto un'enorme espansione dei rapporti di collaborazione a livello internazionale. Grazie all'applicazione della network analysis a fonti informative come il Science Citation

2 Dall'industria dei computer a quella dei semiconduttori, dall'automobile alla chimico-farmaceutica, dall'aerospaziale a quella tessile e meccanica, all'industria dell'alimentazione e all'agro-alimentare. Tra i contributi più significativi si vedano: Ernst e Kim (2002), UNIDO (2004), UNCTAD (2007); Gereffi, (2001), Gereffi et al. (2005); van Raan e Leeuwen (2002); OECD (2007), Morrison et al. (2008). 
Index e a studi bibliometrici condotti con tecniche sempre più sofisticate, sono stati descritti sia campi di ricerca emergenti molto specifici (Shibata et al., 2008), sia la formazione e l'incremento di reti di collaborazioni internazionali in domini molto ampi (Wagner e Leydesdorff, 2008). La dinamica della scienza ha progressivamente assunto la morfologia di un "sistema globale" (Wagner e Leydesdorff, 2006), con architetture regionali inscritte in aggregazioni globali. L'incremento di co-authorships in domini di indagine tecnico-scientifica sempre più dinamici, dato lo sviluppo di una sostenuta competizione tecnico-scientifica, ha messo in luce: 1) Una forte accelerazione nel corso del tempo, nel decennio 1990 e ancor più dopo il 2000. 2) Una crescente densità delle reti, ad indicare un'espansione della connettività tra un numero crescente di protagonisti della ricerca. 3) L'ampliamento del numero di paesi appartenenti ai "gruppi dominanti" ("centrali") delle reti, ma con una significativa dinamica di "esclusioni ed ammissioni", che segnala come nuove entità si affermano mentre altre arretrano. 4) Una dinamica auto-organizzativa delle stesse reti, nella misura in cui i ricercatori e i centri più attivi e creativi hanno esercitato un'attrattività sempre maggiore verso altri partners (Wagner e Leydesdorff, 2005).

Più in generale, poi, le reti di collaborazione scientifica hanno dato luogo a vere e proprie "comunità" dotate, come ha messo in luce Newman $(2001,2004)$, di proprietà topologiche: 1) Esse sono "funzionali" quando hanno la small world property; ciò significa che il numero delle connessioni tra due qualunque entità appartenenti alla rete non supera il numero $6^{3} .2$ ) Spesso mostrano un'elevata densità, stimata attraverso il coefficiente di clustering, che indica strutture connettive intrecciate tra più entità di ricerca. 3) Sono sempre più frequenti comunità con proprietà "ad invarianza di scala", come la "legge di potenza" (scale-free power-law distribution, Barabasi e Albert (1999), detta anche Pareto-distribution), la quale descrive situazioni in cui c'è un numero ristretto di nodi-entità con un numero elevato di connessioni e un numero elevato di nodi con un basso numero di connessioni ${ }^{4}$. Forme evolutive differenti sono rinvenibili in diversi campi scientifici, in base appunto alla ricchezza delle interazioni e delle sfere di conoscenza tra le quali si innescano fecondi processi di cross-fertilization.

Sulla base delle coordinate generali appena descritte appare fondato sostenere che siamo in presenza di un continuo processo di espansione-trasformazione della scienza come sistema all'interno di uno spazio delle conoscenze tecnico-scientifico

\footnotetext{
${ }^{3}$ La small world property è nota anche come six degrees of separation (Watts, 1999).

${ }^{4}$ Tale proprietà viene considerata una sorta di "legge universale", in quanto è verificata in molti domini conoscitivi: biologia (evoluzione genetica di organismi complessi), sviluppo urbano (Zipf's Law), ecologia ed evoluzione di grandi sistemi-reti tecnologiche.
} 
tendenzialmente illimitato. Al suo interno sotto-spazi di ricerca sono individuati ed ampliati, per poi essere circoscritti e quindi trasformati. In un universo della ricerca così caratterizzato la scienza può essere suggestivamente concepita come "mappa" (Fleming e Sorenson, 2001, 2004a,b), che orienta e fornisce strumenti per la dinamica senza fine dell'innovazione, mentre la stessa tecnologia diviene un "sistema complesso adattativo" (Fleming e Sorenson, 2001). È possibile definire a livello teorico e operativo strumenti che consentano di analizzare la propria collocazione rispetto alla "frontiera" tecnico-scientifica ed anche delineare traiettorie evolutive, graduate sulla base della loro probabilità di accadimento, della preferibilità, dei rischi potenziali in esse latenti, e così via. Un'applicazione di questi strumenti la incontreremo nella Terza parte del paper.

In sintesi, il global intelligent space tende ad assumere la natura di sistema complesso (Katz, 2006), incentrato sull'esistenza e l'efficacia di reti innovative con molteplici gradi di complessità, stimata in base alle interazioni che si sviluppano tra molte dimensioni (culturale, istituzionale, tecnico-scientifica, normativa, e così via) ${ }^{5}$. In questo orizzonte generale emerge la quarta macro-tendenza, che sembra mostrare un'evidente accelerazione negli ultimi anni. Intendiamo riferirci al continuo avanzamento della frontiera tecnico-scientifica, con il moltiplicarsi dell'apertura di nuovi domini di indagine e il drastico mutamento di quelli esistenti, fino al punto che, per qualsiasi impresa e centro di ricerca, è ormai impossibile controllare tutte le conoscenze rilevanti per il proprio campo di investigazione. Questa visione si estende anche ai prodotti (beni e servizi), che rappresentano ormai il punto di confluenza di molteplici flussi di conoscenza, i quali a loro volta si sviluppano grazie all'esistenza di dinamiche interattive tra entità che svolgono attività di ricerca in differenti sotto-spazi conoscitivi. L'intersezione -talvolta programmata e altre volte esito di esplorazione più o meno random- tra flussi informativi provenienti da ambiti molteplici è alla base di processi innovativi con due proprietà apparentemente contraddittorie: 1) presenza di traiettorie evolutive sufficientemente stabili, 2) imprevedibilità dei risultati.

Riguardo alla prima proprietà, si pensi ad esempio al profondo cambiamento di paradigma tecno-economico ${ }^{6}$ dal modello basato sulla produzione di massa al modello

\footnotetext{
5 Sull'importanza di fattori come quelli indicati per l'evoluzione di tecnologie complesse la letteratura è naturalmente molto ampia. Per un'analisi accurata di differenti campi tecnico-scientifici e conseguenti prodotti (componenti per turbine, diagnostica cardiologica per immagini, CD, tecnologie per la radioterapia, micro-processori e floppy disk) si veda Rycroft e Kash (2002).

${ }^{6}$ Il concetto di paradigma tecno-economico indica un sistema complesso di elementi che acquisisce una stabilità evolutiva: «This set of interrelated technical and organizational innovations, gradually comes together as a best-practice model or -as we have called it, a techno-economic paradigm"- capable of guiding the diffusion of each specific technological revolution. As it spreads, this new paradigm gradually takes root in collective consciousness, replacing the old ideas and becoming the new "common sense" of
} 
incentrato su reti flessibili; di entrambi Perez (2001) ha esaminato a fondo e le caratteristiche distintive (tipologie di input, assetti organizzativi, quadro istituzionale, sistemi di valori) e le modalità operative (meccanismi di apprendimento e di potere, strumenti per il perseguimento dell'efficienza, e così via). Per quanto concerne la seconda proprietà, ovvero l'imprevedibilità degli esiti di traiettorie di ricerca, essa deriva dal fatto che, grazie alla struttura reticolare e multiscalare, possono realizzarsi processi di combinazione e ri-combinazione di conoscenze, generate in molteplici spazi di ricerca, entro i quali si sviluppano le attività di gruppi di agenti, individuali e collettivi. Per questa via I'universo tecnico-scientifico diviene uno spazio combinatoriale, nel senso che sono tentate e perseguite linee d'indagine con I'elaborazione di concetti nuovi mediante "fusioni" con altri già consolidati'. Tali processi di ibridazione non sono però senza vincoli, dal momento che I"'esplosione combinatoriale" e il connesso pericolo di un caos cognitivo senza punti di approdo sono evitati grazie all'evoluzione dei paradigmi di pensiero, che costituiscono schemi cognitivi di ordinamento della realtà nel corso di processi esplorativi-cognitivi. Nella prospettiva teorica qui delineata stabilità e imprevedibilità sono pertanto proprietà congiunte dell'universo conoscitivo tecnico-scientifico.

Proprio la loro compresenza permette di comprendere la connessione logica tra il quarto macro-trend e il quinto, individuato nelle spinte endogene all'autoorganizzazione dei processi tecnico-scientifici. Quest'ultimo trend si realizza nell'ambito dell'intelligent global space, poiché in esso la ricerca, sviluppata in sottospazi tecnico-scientifici molto differenti e distanti, può innescare fecondi processi di cross-fertilization e quindi di convergenza condizionata verso progetti e beni semplici o complessi $^{8}$. In sostanza, quindi, sono gli spunti teorici precedentemente esposti a fornire le basi cognitive dell'auto-organizzazione dei processi tecnico-scientifici, inducendo a ritenere che questa proprietà non sia occasionale.

\footnotetext{
engineers, managers and investors for the most efficient and "modern" productive practice across the board» (Perez, 2004).

7 Sull'approccio "ricombinante" all'evoluzione della conoscenza tecnologica e scientifica si vedano Weitzman $(1996,1998)$ per una visione standard in termini di funzioni di produzione e Fleming-Sorenson (2001) per un'impostazione evoluzionistica e cognitiva.

${ }^{8}$ In questa visione il processo di produzione può essere concepito come global workspace, al cui interno si sviluppano hierarchical problem solving activities, svolte da individui e organizzazioni attraverso incessanti mappings tra spazi e sotto-spazi conoscitivi. La letteratura in materia, di matrice sia ingegneristica che economico-evoluzionistica, è analizzata in Lombardi (2006, 2008, 2010), dove viene anche sviluppato un approccio morfogenetico alla dinamica organizzativa dei cicli di produzione. Specialmente in Lombardi (2008) vengono analizzati due principi costitutivi dei processi cognitivi umani (composizionalità e ricorsività), che agiscono da schemi ordinatori, in tal modo consentendo di sfuggire periodicamente al rischio di esplosione combinatoriale.
} 


\subsection{Effetti micro, meso e macro-scopici delle traiettorie evolutive nell'intelligent global space}

Nel precedente paragrafo abbiamo tratteggiato i maggiori pattern evolutivi di una dinamica strutturale che ha prodotto e continua a generare effetti molto eterogenei. In questo paragrafo selezioniamo alcuni di tali effetti, distinguendoli sulla base del livello di descrizione.

Effetti micro-scopici: unbundling e rivoluzione algoritmica, nuovi vantaggi competitivi.

Aumentando il livello di granularità dell'analisi, ma sempre in relazione ai macro-trend enunciati, emerge un primo aspetto molto interessante: i processi di produzione, divisi in componenti e sotto-componenti, tendono a divenire sistemi compositi di pacchetti informativi soggetti a incessanti cambiamenti. La combinazione/ricombinazione di conoscenze generate in spazi di ricerca esplorati in qualsiasi parte del mondo è alla base del cosiddetto "secondo unbundling", espressione impiegata per descrivere il fatto che -grazie alla diminuzione dei costi di comunicazione e coordinamento- è venuta meno la necessità di eseguire insiemi compatti di funzioni o fasi manifatturiere. Di qui l'emergere di vari meccanismi di distribuzione a livello internazionale di segmenti oppure di singole componenti dei cicli produttivi, in altre parole processi di «fragmentation, offshoring, vertical specialisation and slicing up the value-added chain» (Baldwin, 2006: 7). Nel nuovo scenario tecnoeconomico mondiale alla competizione per settori e imprese subentra -a partire dall'estremo Oriente- una dinamica competitiva per sotto-insiemi variabili di funzioni economico-produttive ${ }^{9}$. Dalla competizione by sectors and firms si passa a quella by tasks o by production stages, frutto della nuova divisione del lavoro creata dalla possibilità di trading ideas, con l'avvertenza che tale trading va inquadrato in relazione alla creazione di network economico-produttivi globali. Nell'orizzonte descritto cambia profondamente la natura dell'industria manifatturiera, nella misura in cui nel ciclo di produzione le funzioni terziarie si integrano sempre più con quelle più prettamente produttive, dato il rilievo assunto dalla generazione e diffusione di conoscenze in ogni

\footnotetext{
9 Traduciamo con "sotto-insiemi variabili di funzioni economico-produttive" I'espressione inglese packages of tasks (Baldwin, 2006: 24) per indicare che alla base vi sono flussi incessanti di persone, merci e informazioni (idee). Su queste basi l'allocazione di sotto-insiemi di attività a scala globale è espressione della ricerca incessante di miglioramenti delle conoscenze esistenti e di scoperta di nuove, appunto ciò che abbiamo definito "esplorazione di sotto-spazi nell'ambito di uno spazio globale di lavoro". Per un'analisi del secondo unbundling in riferimento ai beni intermedi si veda Yi (2003). Questi temi sono discussi in Lombardi e Macchi (2010) nella prospettiva degli scenari che si aprono alle economie dei Paesi in via si sviluppo.
} 
fase della sequenza che va dall'ideazione di un bene alla sua realizzazione e alla sua proiezione verso il mercato. Più in generale, le "infrastrutture immateriali" della dinamica economica divengono parte essenziale di un sistema produttivo complesso: ideazione e sviluppo delle conoscenze, formazione di risorse umane, disponibilità di quelle materiali e finanziarie.

Queste tendenze investono in modo diretto anche i servizi, nei quali è da alcuni anni in atto ciò che è stata definita "rivoluzione algoritmica" (Zysman, 2006). Essa denota il processo di trasformazione progressiva di molte attività terziarie in sequenze basate su regole formalizzate, codificate e rese computabili, che poi sono riprogrammabili in funzione delle esigenze e dei problemi da risolvere. I servizi tendono a diventare combinazioni mutevoli di conoscenza codificata e tacita, a seconda delle interazioni user-producer, nonché dei cicli di feedback tra agenti (individui e organizzazioni). Su queste basi la stessa impresa si trasforma profondamente: diviene una gerarchia distribuita di attività, compiti, funzioni, obiettivi e disegni strategici (Rouse e Baba, 2006), in una configurazione a network dove il mix variabile di "legami forti" e "legami deboli" è alla base della sua capacità di intercettare flussi informativi e quindi di partecipare al processo innovativo. Un'entità con tali caratteristiche, ovvero composta di gruppi semi-autonomi, richiede lo svolgimento di funzioni di coordinamento, che a loro volta dipendono dall'operatività di "tecnologie intelligenti" e dalla disponibilità di capitale umano in grado di combinare gli input in modo da generare risposte adattative ai cambiamenti.

Strettamente connessi all'unbundling e alla "rivoluzione algoritmica" sono i cambiamenti intervenuti nei vantaggi competitivi, oggi non più perseguibili da una singola impresa, bensì da aggregati multiformi di esse a varia scala, a seconda delle funzioni economico-produttive o dei segmenti di esse, sui quali i SSL sviluppano competenze dinamiche. Torneremo su questo nella Seconda parte del paper.

Effetti meso-scopici: evoluzione dei sistemi socio-economici locali, cambiamenti di basilari proprietà sistemiche

Fino a qualche anno fa i SSL sono stati pensati tramite alcune peculiarità, che da un punto di vista sistemico possono essere così descritte.

\section{Auto-contenimento ${ }^{10}$}

\footnotetext{
10 Per auto-contenimento si intende indipendenza relativa nello svolgimento di una funzione o di un gruppo di funzioni: «un'unità è autocontenuta nella misura in cui le condizioni per lo svolgimento delle sue attività sono indipendenti da ciò che viene eseguito in altre unità organizzative» (March - Simon, 1958: 28).
} 
Il concetto sintetizza alcuni fattori ritenuti basilari dalla letteratura. A) Comunanza dei valori in una determinata area. 2) Ambiti di esplicazione delle relazioni di competitività e cooperazione. C) Modalità di orientamento e tipologia dei flussi informativi. D) Visibilità esterno-interno.

L'autocontenimento è una proprietà sistemica che racchiude molte componenti sulla base della configurazione spaziale e culturale (in senso lato) delle unità coinvolte in un ciclo produttivo; nel caso del "distretto industriale", ad esempio, esso assume una funzione cruciale proprio nel determinare una limitata autoreferenzialità di determinati microuniversi locali. Nei distretti industriali i rapporti di competizione e di cooperazione tra unità si sviluppano sulla base di vincoli determinati anche dal tipo di informazioni che circolano nel sistema. Poiché le informazioni di mercato possedute dagli imprenditori terminali sono rilevanti per tutto il sistema ${ }^{11}$, la dinamica infradistrettuale è di tipo prevalentemente tecnico-produttiva e favorisce processi d'innovazione prevalentemente incrementale e diffusa (Bellandi, 1989). Le fonti endogene delle conoscenze, i peculiari meccanismi di trasmissione (relazioni personali, "atmosfera industriale") determinano una moderata flessibilità nella ricezione dei flussi informativi esogeni (Becattini, 1991).

\section{Gerarchia informativa}

Essa è basata sulla distinzione tra informazioni tecnico-produttive e quelle relative ai mercati. Le seconde sono possedute dall'"imprenditore terminale", che svolge funzioni essenziali di conversione dei segnali percepiti dal mercato in ordinicommesse per le unità operative interne al distretto. Queste ultime sono scelte in base alle connessioni esistenti e attivabili tra le entità distrettuali e lo stesso imprenditore terminale, la cui funzione è cruciale nel sollecitare le potenzialità esistenti ${ }^{12}$. Due sono pertanto i livelli di informazione: i flussi informativi verso l'esterno sono "formalizzati" e controllati, mentre quelli verso I'interno sono informali e ad elevata fluidità diffusiva. Per questa via si crea una sorta di barriera in termini di

\footnotetext{
${ }^{11}$ I legami forti favoriscono attività di exploitation delle conoscenze esistenti, ovvero di adattamento e sviluppo degli stili di pensiero consolidati. In altri termini non si fuoriesce da spazi conoscitivi con limiti definiti. I legami deboli implicano la possibilità di accedere a spazi conoscitivi "Iontani", esplorati da altre entità della rete, con cui possono vengono scambiati acquisizioni conoscitive. La distinzione tra exploitation and exploration, applicate originariamente alla teoria dell'impresa è di March (1991) e successivamente estese alla morfologia evolutiva di imprese e assetti spaziali (Ozman, 2009). Si veda oltre il riferimento a Granovetter e alle tipologie di legami (forti e deboli).

12 L'imprenditore terminale «da un lato osserva attentamente le vicende del mercato mondiale dei prodotti del distretto e dall'altro approfondisce in continuazione la sua conoscenza del distretto come entità produttiva e socio-culturale. La sua funzione specifica sta nel tradurre in termini di prodotti vendibili su quel mercato tutte le potenzialità racchiuse ne tessuto storico del distretto» (Becattini, 1991: $57)$.
} 
visibilità tra ambiente interno ai SSL e quello esterno, i cui input verso il contesto locale sono prevalentemente "filtrati".

\section{Flessibilità adattativa}

Un SSL dalla forma distrettuale appare particolarmente adatto a risolvere problemi tecnico-produttivi in risposta a parametri esogeni (tecnologici, di mercato). In esso infatti l'elaborazione distribuita e parallela delle informazioni si traduce in un sistema organizzato in moduli flessibili, fortemente interattivi tra loro e in grado di individuare e selezionare comportamenti appropriati per i parametri della domanda finale. In concreto, le piccole imprese localizzate in un dato territorio costituiscono gruppi di fasi stratificate all'interno della sequenza complessiva di produzione. Esse incrementano, accumulano e migliorano le proprie competenze nell'attività di risoluzione dei problemi, ovvero nel perseguire l'obiettivo di sintonizzare la capacità produttiva sui parametri della domanda. Le unità produttive possono essere quindi considerate agenti adattativi, con comportamenti goal oriented, che sviluppano competenze complementari, omogenee ed anche alternative, mediante continue interazioni. Mentre ciò avviene sulla base di interazioni ad alta frequenza tra una miriade di unità, le interazioni "a bassa frequenza" con l'ambiente esterno spettano in esclusiva agli imprenditori terminali, definiti in letteratura come veri e propri gatekeeper informativi.

Le quattro proprietà sistemiche sono erose e sostanzialmente trasformate dalla dinamica tracciata nel precedente paragrafo, che modifica almeno tre ambiti strutturali dei SSL: A) distinzione tra milieu interno e ambiente esterno, con conseguenti nuove modalità di interazione con i mercati e la dinamica tecnologica, sulla base di non tradizionali meccanismi di acquisizione e elaborazione delle informazioni. B) Nuova divisione cognitiva del lavoro e pluralità di modelli tecnicoorganizzativi all'interno delle agglomerazioni locali. C) Formazione e consolidamento di reti di imprese organizzate in forme gerarchiche tendenzialmente globali.

In questa prospettiva l'analisi della dimensione territoriale dei processi socioeconomici richiede l'adozione di una prospettiva relazionale e topologica (Amin, 2002). Il matching strategico tra potenzialità locali e reti globali diviene un ambito decisivo di studio, in quanto le interdipendenze sono l'ingrediente essenziale di processi multiscalari, dove intervengono agenti molteplici (imprese, organizzazioni, istituzioni) in mix variabili di cooperazione e competizione (Coe et al., 2004; Ernst e Kim, 2002). Lo spazio tecno-economico che viene esplorato dipende dalla formazione di competenze dinamiche localizzate, decisive per "assorbire" flussi tecno-economici a scala globale. 
La dinamica reticolare multi-scalare assume dunque una centralità senza precedenti, con alcune profonde implicazioni: 1) Molti network possono e forse debbono sovrapporsi nei SSL, alla ricerca di risorse immateriali ivi esistenti. 2) L'appartenenza a network condiziona in modo decisivo l'evoluzione di imprese e SSL, perché da essa dipendono forme e tipologie di apprendimento. 3) La posizione occupata all'interno degli stessi network influenza il dinamismo evolutivo, dal momento il tipo di legami che istituiscono (se di tipo "forte" oppure "debole" à la Granovetter, 1973) condiziona l'accesso ai flussi di informazione e conoscenza: i primi favoriscono processi conoscitivi di tipo incrementale, i secondi consentono di accedere a flussi di conoscenze innovative ${ }^{13}$.

Effetti macroscopici: dinamica incentrata su sistemi socio-tecnici multi-livello e multiscala

Le trasformazioni strutturali degli ultimi decenni, sopra brevemente ricostruite, si incentrano su sistemi socio-tecnici che evolvono ${ }^{14}$.

Il punto cruciale ai fini della nostra trattazione è che siamo in presenza di sistemi multi-livello e multi-scalari. Nucleo teorico fondante del concetto di sistema socio-tecnico è il "regime tecnologico" (Kemp, Schot Hoogma, 1998; Geels e Kemp, 2007), interpretato come insieme di regole stratificate, le quali strutturano le modalità con cui si affrontano i problemi tecnico-produttivi e le soluzioni operative adottate, che a loro volta hanno una duplice valenza: 1) si incorporano nella pratica di scienziati, tecnici e imprese, 2) dipendono dall'interazione complessa tra varie sfere della vita socio-economica (culturale, normativo, istituzionale), a differenti livelli (dal locale alla dimensione internazionale).

\footnotetext{
13 I legami forti favoriscono attività di exploitation delle conoscenze esistenti, ovvero di adattamento e sviluppo degli stili di pensiero consolidati. In altri termini non si fuoriesce da spazi conoscitivi con limiti definiti. I legami deboli implicano la possibilità di accedere a spazi conoscitivi "Iontani", esplorati da altre entità della rete, con cui possono vengono scambiati acquisizioni conoscitive. La distinzione tra exploitation and exploration, applicate originariamente alla teoria dell'impresa è di March (1991) e successivamente estese alla morfologia evolutiva di imprese e assetti spaziali (Ozman, 2009)

${ }^{14}$ L'espressione sistemi socio-tecnici designa le combinazioni di large technological systems nell'ambito di regimi tecnologici, regimi socio-tecnici e scenari socio-tecnici (socio-technical landascapes). Questi ultimi sono le rappresentazioni a livello macro di combinazioni di variabili: prezzi e quantità degli input energetici, fattori di crescita a livello internazionale, eventi socio-politici, questioni ambientali, innovazioni tecnologiche radicali, e così via. In breve, è l'insieme di componenti endogene e soprattutto esogene che influenzano lo scenario entro cui gli agenti operano. Saggi essenziali di riferimento sono: Hughes (1987), Constant II (1987), Van de Poel (2003), Markard e Truffer (2008), Geels (2004), Verbong et al. (2008).
} 


\subsection{SinTESI}

Nell'odierno contesto il cambiamento dei SSL è profondamente connesso alla dinamica di sistemi socio-tecnici, il cui profilo evolve incessantemente in conseguenza di processi multi-scalari e multi-livello, che permeano le cinque traiettorie descritte nelle pagine iniziali. La vitalità e le probabilità di successo dei SSL sono correlate al grado e alle modalità di inserimento negli scenari socio-tecnici, tenendo presente che oggi più che in passato esse dipendono dalla capacità di elaborazione strategica nella ricerca di una collocazione all'interno dell'intelligent global space.

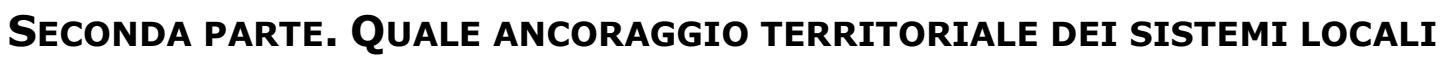

\subsection{SISTEMI SOCIO-ECONOMICI LOCALI SONO SOLTANTO "LOCALI"?}

Dopo aver esaminato gli effetti micro, meso e macro-scopici delle traiettorie evolutive nell'intelligent global space, volgiamoci a considerare che cosa non può non cambiare nella rappresentazione teorica dei SSL.

Qualora volessimo caratterizzare in termini molto generali uno degli elementi più rilevanti dell'ampio dibattito sui SSL, I'attenzione non può che mettere al centro il loro ancoraggio territoriale (Hamdouch, 2009). Da una parte è stato infatti elaborato un paradigma che enfatizza il ruolo delle economie esterne alle singole imprese ma interne ai luoghi del SSL: esse comprendono soprattutto le economie di specializzazione, di apprendimento, d'imprenditorialità diffusa e di costi di transazione (Dei Ottati, 2009). Dall'altra parte si è ampliato lo schema interpretativo, affiancando all'analisi della prossimità geografica - che si riferisce alla distanza fisica tra due imprese, ovvero al tempo di spostamento dall'una all'altra - una pari attenzione verso la prossimità cognitiva, se le imprese condividono la stessa base di conoscenze; verso quella organizzativa, se stanno sotto un comune controllo gerarchico; verso quella sociale, se i loro membri hanno relazioni amichevoli; e verso quella istituzionale, se operano entro le medesime istituzioni (Boschma, 2005). Il denominatore comune che distingue le ultime quattro modalità dalla prossimità geografica, è che esse esprimono tutte una prossimità relazionale in cui l'interazione dei soggetti (individuali 0 organizzati) può fluire indipendentemente dalla loro vicinanza fisica (Amin-Cohendet, 
2003). Ne segue che la riproduzione del SSL, richiedendo varie forme di prossimità tra gli attori, avviene su più livelli strutturali e su una molteplicità di scale dimensionali. Questo spessore multidimensionale del SSL è stato altrettanto efficacemente concettualizzato distinguendo tre tipi di "immersione" (embedding) delle attività economiche nelle relazioni sociali: mentre il tipo chiamato "istituzionale" è spesso localizzato, quello "strutturale" si riferisce ai network in cui il SSL è inserito, e quello "relazionale" alla natura e all'intensità dei legami che i soggetti del SSL hanno nei network (Nooteboom, 2004). Ma un network può sia avere una collocazione territoriale, sia non averne, e lo stesso vale per i legami che esso contiene. Nulla quindi impedisce al SSL di evolvere con forme di radicamento sociale che si sganciano da un preciso territorio.

Il punto di vista dell'ancoraggio territoriale ha prevalso lungamente, sia perché rappresentativo del senso comune (il "locale" coincide con il luogo, ovvio!), sia perché riposa sull'autorevolezza degli studiosi di riferimento (Marshall, Krugman, Porter) ${ }^{15}$, secondo i quali sono in definitiva le agglomerazioni spaziali e l'apprendimento sulla base di scambi conoscitivi interpersonali a rendere possibile, sotto determinate condizioni, i vantaggi competitivi di un contesto territoriale rispetto ad un altro. La dinamiche di apprendimento sono correlate alle interdipendenze tra agenti che si sviluppano a scala territoriale, in genere locale o regionale; entro quella scala si individuano attori centrali (le interfacce con l'esterno, gli intermediari, gli imprenditori finali, e così via); anche quando si ammette che altre scale sono di rilievo per il successo del SSL, non si assume quale problema teorico l'articolazione delle differenti scale spaziali (Hamdouch-Depret, 2009).

L'altro punto di vista è meno intuitivo, perché la collaborazione tra i soggetti (individuali o collettivi) corre lungo coordinate geografiche, cognitive, organizzative, sociali e istituzionali, essa può realizzarsi in una pluralità di luoghi e di dimensioni. Un SSL trae forza dalla sua capacità di ibridare le conoscenze approntate localmente con quelle assorbite altrove, dall'efficacia nel combinare lo sfruttamento degli asset specifici generati internamente con l'esplorazione - favorita da legami deboli extralocali - di sentieri tecnico-organizzativi avviati da altri SSL. Il SSL s'irrobustisce e dinamicizza ancorandosi a parecchi territori, connotati da diverse strategie, politiche e risorse. Se dunque, per un verso, un SSL è immerso in network locali, i suoi luoghi sono, per l'altro verso, immersi in network multi-scala. Soltanto rendendosi

${ }^{15}$ Si veda Marshall (1890), Krugman (1991); Porter (1990). 
permeabile a logiche innovative plurali che trascendono i suoi confini geografici, un SSL può co-adattarsi e co-innovare.

Riprendendo alcune tematiche della parte iniziale del paper, ci proponiamo di esplicitare alcune implicazioni teoriche della seconda prospettiva.

\subsection{DE-VERTICALIZZAZIONE E TASK-BASED PRODUCTION}

Come ricordato, a partire dagli anni '80 i processi di produzione vengono frammentati in un elevato numero di componenti (tasks) che possono essere separate nel tempo e nello spazio (Grossman - Rossi-Hansberg 2006). Ne segue che il commercio internazionale manifatturiero e progressivamente anche quello delle attività terziarie mostrano un'organizzazione produttiva del tutto nuova: da flusso continuo e verticalmente integrato in una medesima localizzazione si passa allo sviluppo di funzioni di coordinamento globale tra una molteplicità variabile di attività parcellizzate (Baldwin, 2006).

Nella misura in cui si realizza la scomposizione planetaria dei cicli produttivi, cambia la natura stessa della competizione tra SSL. Prima essa avveniva tra territori in grado di esprimere agglomerazioni di imprese specializzate nella produzione di determinati beni finali. Adesso tende a verificarsi tra territori dotati di capabilities dinamiche rilevanti per lo svolgimento di determinati tasks. Se nel passato erano le imprese ad essere "specificamente situate", socialmente costruite e localmente integrate, attualmente il radicamento territoriale, che pure rimane importante, riguarda prevalentemente le task capabilities, le quali non possono formarsi sempre e ovunque, perché richiedono una particolare combinazione di input specifici.

La scomposizione di fasi del ciclo produttivo e la loro distribuzione a livello internazionale non sono peraltro limitate alla manifattura dei beni tangibili, ma riguardano anche la formazione della conoscenza occorrente per definire e realizzare nuove generazioni di beni. L'innovazione è oggi sempre più il risultato dell'impegno congiunto di più organizzazioni. Ne segue che i centri di ricerca centralizzati nelle imprese più grandi hanno una rilevanza decrescente, a favore di consorzi ad hoc tra imprese di varie dimensioni, spesso sostenuti anche da finanziamenti pubblici. Questi consorzi - per esprimerci intanto con una formulazione provvisoria su cui torneremo coltivano e valorizzano gruppi di task capabilities tra loro complementari (Rosenbloom e Spencer, 1996). 
Secondo la teoria standard, più precisamente nella versione rappresentata dall'"economia dei costi di transazione", processi di integrazione verticale delle imprese si realizzano in condizioni di razionalità limitata, incompletezza contrattuale, incertezza e specificità degli investimenti. In simili contesti, infatti, la struttura di governance alternativa all'impiego di meccanismi contrattuali di mercato consente il perseguimento di scelte ottimali nel fronteggiare i problemi posti dall'asimmetria informativa tra gli agenti e dai costi di transazione (Roberts, 2004). La deverticalizzazione dell'impresa e il ricorso a strumenti contrattuali si ha quando le caratteristiche in questione sono ridotte oppure assenti. La teoria dei costi di transazione ha dato luogo ad un vasto dibattito, ancora in corso; lungi quindi da noi I'idea di voler sintetizzare la materia e quindi chiudere la discussione. In questa sede ci limitiamo a riprendere uno dei temi più interessanti, emersi nel corso del confronto teorico ed empirico tra studiosi. Un numero crescente di questi sottolinea infatti che, in condizioni di profonda incertezza di fonte esogena ed endogena ${ }^{16}$, le imprese diventano incapaci di specificare ex ante le caratteristiche di funzionamento del bene che produrranno. In simili circostanze appaiono inadeguati tanto i contratti formali quali sistemi di vincoli e incentivi tali da orientare le parti a mantenere determinati comportamenti - quanto gli accordi informali - la cui efficacia dipende da pre-esistenti livelli di fiducia. Tendono piuttosto ad affiorare meccanismi pragmatici di coordinamento tra imprese con capacità diverse ma complementari, mediante cui le parti esplorano congiuntamente la progettazione, la produzione e le endemiche ambiguità organizzative delle attività economiche innovative. Questi co-design contracts, pur mobilitando investimenti relation-specific, riducono le tentazioni opportunistiche di fuga mediante intrecci di contrattazioni formali e negoziazioni informali. Mentre nessun impegno singolo è di per sé stringente, l'intreccio come tale compone un processo iterativo lungo cui aumentano i costi di transizione (switching costs) - gli oneri del passaggio ad un assetto organizzativo con differenti partner - , rendendo conveniente proseguire la collaborazione. Si tratta di una configurazione che facilita l'apprendimento cooperativo in modi da un lato più formali rispetto a quelli fluidi e quasi spontanei del classico distretto o cluster, e dall'altro più informali rispetto agli ordinamenti gerarchici (Gilson et al., 2009).

Questi consorzi semi-stabili e semi-istituzionalizzati non richiedono necessariamente la contiguità spaziale dei membri e possono includere, accanto alle

\footnotetext{
${ }^{16}$ La fonte è endogena quando si sviluppa all'interno di processi esterni ai processi economici in cui la stessa impresa è impegnata; l'esogenità si ha invece allorché fattori e variabili non previsti e non prevedibili (innovazioni radicali, macro-invenzioni) cambiano il quadro concettuale di fondo del mondo tecnico-scientifico.
} 
imprese, numerose altre tipologie di soggetti economici, a cominciare dai consumatori. Il concetto di "apprendimento trialogico" è stato elaborato proprio per rappresentare simili interazioni creative prive di contiguità tra agenti eterogenei. Siamo abituati all'apprendimento monologico, che si svolge entro una mente singola, oppure a quello dialogico, che si effettua tra più persone ma richiede un comune linguaggio, regole approvate e sperimentate, obiettivi espliciti e convergenti. Nel "trialogo" realizziamo piuttosto un'interazione collaborativa mediante la formazione e l'implementazione di un artefatto concettuale, di una pratica sociale o di un prodotto economico. Mentre dunque il dialogo presuppone un basamento condiviso per rendere possibile la comunicazione, nel trialogo i processi sono così il mezzo come il fine della connessione e comportano la co-evoluzione di chi è in essi coinvolto (PaavolaHakkarainen, 2005) ${ }^{17}$.

Ai nostri fini sembra importante sottolineare che si vanno moltiplicando gli spazi cognitivi complessi, dove assumono centralità le strutture interattive tra agenti (individuali e collettivi), i quali attraverso di esse incessantemente sviluppano, modificano, trasformano i propri contesti e processi cognitivi. Analogamente, numerosi SSL affiancano al loro ancoraggio locale forme di apprendimento trialogico in cui contano meno sia la convergenza "comunitaria" su valori e procedure, sia la distanza spazio-temporale.

\subsection{VARIETÀ CORRELATE E TRAIETTORIE DISCONTINUE}

Alcuni modi tradizionali d'indagine dello sviluppo e dell'innovazione dei SSL consistono nel mettere a fuoco l'intensità fattoriale oppure le differenze tecnologiche. La prima prospettiva suggerisce che il sentiero di crescita e cambiamento di un SSL dipende dalle proporzioni relative degli input posseduti e dalle proporzioni in cui tali input occorrono per produrre i vari beni. L'altro punto di vista sostiene che, dato uno spazio tecnologico, il progresso si realizza a misura che un SSL produce beni che usano tecniche vicine alla frontiera mobile di quello spazio.

\footnotetext{
17 Non ci pare infondato inquadrare nello schema trialogico uno dei "fenomeni" più discussi nella nostra knowledge-based society. Intendiamo riferirci a Wikipedia. I suoi percorsi infiniti di riscrittura degli articoli dell'enciclopedia sono propriamente ciò che sollecita l'impegno di tante persone in tanti luoghi, e sono ciò che unisce e conferisce senso a quegli sforzi. Wikipedia non può operare come un sistema dai confini ben delimitati, bensì si afferma aprendosi ad altri sistemi cognitivi ed organizzativi tramite nessi sia competitivi che reticolari. Essa è multi-territorializzata, poiché riceve contributi da tanti luoghi, e multilivello, poiché funziona su varie scale e con vari gradi gerarchici. In tal senso qualche studioso inizia a definirlo in termini di global mind, ma è bene trattare i temi a riguardo in altra sede.
} 
Una differente prospettiva è stata introdotta recentemente da Ricardo Hausmann e colleghi (Hidalgo et al., 2007). Essa enfatizza l'importanza del reticolo di nessi in cui ciascun task, oppure ciascun bene, è immerso: a monte e a valle (in una matrice input-output), orizzontali (se sono tasks o beni complementari) e trasversali (se richiedono beni pubblici, quali ad esempio istituzioni, infrastrutture o culture condivise $)^{18}$. Per i nostri scopi espositivi, questa teoria aiuta a meglio indagare due aspetti finora trascurati. Anzitutto, considerando un prodotto rispetto agli altri prodotti, segnala che un SSL deve evitare tanto la trappola dell'iper-diversificazione quanto quella della iper-specializzazione. Se infatti esso presentasse una differenziazione molto elevata della struttura produttiva, avrebbe una ridotta prossimità cognitiva tra le industrie e una scarsa possibilità di istituire percorsi di apprendimento collettivo e di innovazione collaborativa come quelli richiamati nel $\S 2.2$. Se al contrario fosse rigidamente legato a pochi settori, sarebbe molto bassa la sua capacità di perseguire idee nuove e di cambiare traiettoria socio-tecnica. Sono dunque d'importanza strategica le "varietà correlate" (related variety) tra settori, ossia il loro essere collegati in termini di condivisione o complementarità delle conoscenze di base e delle competenze (Frenken et al., 2007). Tali varietà permettono di spostare gli investimenti specifici da un'attività ad una "prossima", senza che essi perdano troppo valore: si tratta infatti di concepire il cambiamento di un'attività produttiva in termini di passaggio da uno spazio cognitivo (di natura tecnicoproduttiva) ad un altro, tenendo presente che l'intero spazio è soggetto a processi evolutivi differenti per intensità e livello. Ad esempio, un tipo di mutamento di minore pregnanza è il seguente: le risorse necessarie per produrre magliette di cotone sono probabilmente trasferibili in maniera meno onerosa verso la produzione di magliette di lana che non in quella di computer. Ben differente è il caso in cui si debba passare dalla produzione di tessuti tradizionali alla produzione con agenti naturali oppure alla produzione di tessuti che elaborano informazioni. Qui siamo difatti in presenza di una transizione tra differenti regimi e sistemi socio-tecnici, tale da richiedere un mutamento basilare degli input tecnico-scientifici e delle funzioni di coordinamento tra fasi.

\footnotetext{
${ }^{18}$ In termini di misurazione, questa teoria stabilisce una misura della distanza, denominata 'prossimità', tra una coppia di beni, basata sulla probabilità che entrambi siano esportati dagli stessi SSL. Tale misura si basa sui RCA (revealed competitive advantages) negli scambi internazionali, i quali calcolano il rapporto tra l'esportazione del bene $x$ di un SSL e il suo export totale, rispetto al medesimo rapporto di un SSL medio. Infine, estende tale procedimento a tutte le coppie di beni, ottenendo il reticolo globale. I dati per elaborare l'intero reticolo sono già dettagliati per 132 Paesi in http://www.chidalgo.com/productspace/index.htm. Per un'applicazione all'Italia, si veda Lombardi et al. (2009, nota 23).
} 
Gli input altamente specifici - ovvero le capabilities umane, fisiche e istituzionali localmente disponibili - danno forma ad un task o ad un bene che, in quanto li utilizza per la propria produzione, è pure altamente specifico. Il 'livello di sofisticazione' di un prodotto dipende dal numero e dalla tipologia di capabilities che esso richiede, mentre il 'livello di complessità' di un SSL è correlato all'insieme di capabilities territorialmente accessibile.

L'approccio di Hausmann e colleghi dedica inoltre attenzione alle discontinuità. Ogni SSL tende anzitutto ad "evolvere" spostandosi verso la produzione di tasks o beni che sono, nel reticolo, vicini ai tasks o ai beni che esso è già in grado di produrre/esportare, ossia tende a valorizzare ${ }^{19}$ le proprie capabilities, rafforzando I'esistente pattern di specializzazione. D'altro canto un SSL può "svilupparsi" quando riesce a cambiare traiettoria, ovvero a saltare da un'area ad un'altra - da un nodo ad un altro - nello spazio mondiale dei prodotti, malgrado sia elevata la distanza tra i prodotti situati nelle due aree ${ }^{20}$. «Immaginiamo che il prodotto sia un albero e che l'insieme dei prodotti sia la foresta. Un [SSL] è composto da una costellazione d'imprese, ovvero di scimmie che vivono sugli alberi usando i differenti prodotti. Il processo di crescita comporta lo spostamento dalla parte più povera della foresta, dove gli alberi hanno pochi frutti, verso le parti migliori. Ciò comporta che le scimmie debbano saltare da un albero all'altro, ossia debbono ricostituire il capitale (fisico, umano e istituzionale) per prodotti differenti da quelli fino ad allora prodotti. La tradizionale teoria della crescita assume che vi sia sempre un albero a portata di mano, e che quindi la struttura della foresta non è importante. Se però la foresta è eterogenea, con alcune zone dense e altre quasi desertiche, e se le scimmie possono saltare soltanto limitate distanze, allora le scimmie possono essere incapaci di spostarsi lungo tutta la foresta. Se accade questo, la struttura dello spazio, e la posizione del [SSL] in esso, diventano del massimo rilievo» (Hildago et al., 2007: $482)^{21}$.

Va rimarcato che questo approccio poggia sulla teoria delle reti, la quale, come già ricordato nella Prima parte, ha mostrato come esse abbiano la tendenza ad organizzarsi attorno a un'architettura estremamente concentrata, mentre la distribuzione dei collegamenti tra i nodi tende verso una struttura "ad invarianza di

\footnotetext{
${ }^{19}$ Il termine va inteso nel senso di ampliamento e diversificazione delle capabilities dinamiche.

20 Brasile, Indonesia e Turchia sono paesi che, partendo dalla produzione ed esportazione di beni poco sofisticati, hanno rapidamente realizzato una trasformazione strutturale. Si veda Hidalgo (2009).

${ }^{21}$ Possiamo misurare la prossimità media (o 'densità') di un nuovo bene potenziale per l'attuale struttura produttiva di un SSL e, per esaminare la traiettoria del vantaggio comparato di un SSL, possiamo concentrarci sui 'beni in transizione', ossia su quelli che presentano un passaggio, in un tempo di cinque anni, del RCA tra una certa coppia di beni da valori inferiori a 0,5 a valori superiori all'unità.
} 
scala" (o legge di potenza): in molti campi la topologia delle reti, ossia la configurazione delle relazioni tra nodi-entità, non è casuale (ciascun nodo si connette con qualsiasi altro), ma segue una ben determinata distribuzione di probabilità, che è invariante rispetto alla scala del fenomeno indagato (sia esso una rete metabolica, un ecosistema, una configurazione genetica). Ciò accade perché la probabilità di connessioni tra nodi in una rete segue appunto la "legge di potenza": chi si connette ad una rete tende a collegarsi ai nodi con un maggior numero di connessioni ${ }^{22}$. $\mathrm{E}^{\prime}$ bene precisare che gli autori in questione individuano questa legge, ma ciò non implica che siano escluse altre morfologie, anzi la varietà morfologica è essenziale per la dinamica delle reti, specie se basate su flussi informativi e conoscitivi.

Riprendendo in esame il modello di Hidalgo et al (2007), una sua implicazione rilevante è la tendenziale polarizzazione dello spazio mondiale dei prodotti, al cui interno si producono incessantemente asimmetrie. La proprietà topologica di cui gode tale spazio $^{23}$ fa sì che sia possibile spostarsi al suo interno grazie a pochissime connessioni. Tale impostazione teorica appare particolarmente suggestiva ed efficace, perché consente di rappresentare i sistemi e i cicli produttivi a livello internazionale come spazio tecnico-produttivo delle interrelazioni tra unità-nodi. In questa prospettiva sia l'evoluzione graduale di un SSL che il suo sviluppo discontinuo sono resi possibili dalla posizione del sotto-spazio che esso occupa: alcune aree facilitano upgrading verso aree del reticolo ad elevato numero di connessioni; altre meno. Gli spostamenti possono essere in qualche modo 'previsti' e in un certo senso oggetto di strategie, in quanto i SSL tendono a convergere nel reticolo globale verso le posizioni e i connessi livelli di reddito associati alla complessità della propria struttura produttiva (Hidalgo et al., 2007). Rovesciando la prospettiva, può accadere che SSL con livelli simili di produzione e di sofisticatezza dei tasks o dei beni esportati abbiano significative variazioni nel set di alternative implicate dalla loro attuale struttura produttiva, cosicché soltanto alcuni di loro manterranno traiettorie vitali di trasformazione strutturale.

Nella teoria di Hausmann e colleghi lo spazio è quindi un insieme di potenzialità e di vincoli. Se torniamo alla metafora della foresta, le imprese-scimmie desiderano produrre un dato task o bene, ovvero usare il tipo di frutta generato da un certo

\footnotetext{
22 E' basilare il concetto di preferential attachment, proposto nel famoso contributo di Barabasi e Albert (1999), che è considerato il punto di innesco di questo filone di ricerca. Su di esso si basa la formulazione della legge secondo cui la probabilità che in una rete un nodo sia connesso con $k$ vertici è rappresentata dalla funzione $k$ elevata ad un parametro negativo (stime di tale parametro variano in relazione ai campi di indagine). Tale parametro è invariante rispetto alla scala dei fenomeni e processi indagati.

${ }^{23}$ In estrema sintesi: la legge di potenza significa che vi sono pochi nodi con un numero elevato di connessioni e moltissimi con un numero esiguo.
} 
albero. Se la foresta fosse un luogo omogeneo con costi di spostamento nulli, in linea di principio qualsiasi albero potrebbe generare qualsiasi frutto. Se di fatto così non avviene, è soltanto perché (sostiene la teoria tradizionale) varia la redditività relativa degli inputs da un albero all'altro e quindi alle banane saranno dedicati gli alberi più produttivi nel combinare gli inputs occorrenti, mentre altri alberi renderanno di più combinando gli ingredienti che danno i fichi o le mandorle. La teoria di Hausmann e colleghi critica ed empiricamente smentisce questa prospettiva. Ogni frutto scaturisce da un albero in cui sono decisive le varietà correlate delle industrie e le capabilities "localmente situate". Pertanto non ovunque, nella foresta, potremmo raccogliere banane $\mathrm{e}$, in questo senso, lo spazio è ancora territorialmente ancorato. D'altra parte esistono circostanze, assai rilevanti proprio perché meno frequenti, in cui lo spazio mondiale dei prodotti si deforma e comprime, consentendo all'impresa-scimmia di saltare in una zona diversa della foresta. A sua volta, I'analisi delle "varietà correlate" ci suggerisce che flussi esterni di conoscenza e di risorse, pur preziosi, possono modificare poco il SSL se presentano una prossimità cognitiva troppo bassa, ovvero se si staccano eccessivamente dalle competenze e dalle conoscenze di base ivi operanti (si veda Boschma-Iammarino, 2009, per una conferma nel caso italiano). Per questi motivi nelle fasi di trasformazione tecno-economica è essenziale che gli agenti (i SSL tra essi) amplino la gamma delle opzioni strategiche possibili attraverso l'espansione del proprio spazio cognitivo e tecnico-produttivo, ad esempio attraverso gli innesti consentiti da "legami deboli". Altrimenti diviene elevato il rischio di marginalizzazione, auto-referenzialità e quindi di implosione, dovuti al fatto di essere "imprigionati" in un sotto-spazio in via di esaurimento.

Dalle riflessioni svolte può essere desunta una proposizione interessante: per comprendere $\mathrm{i}$ fattori e processi che consentono ad alcuni SSL di ottenere migliori performances, occorre analizzare lo spazio mondiale dei prodotti come insieme di potenzialità e di vincoli. Ciò implica che esso non è né piatto né uniforme, ma può contrarsi all'improvviso, e soprattutto che si basa sulle connessioni tra attività e funzioni tecno-economiche. $E^{\prime}$ da esclude che tale spazio sia un'area vuota in cui riversare a piacimento qualsiasi contenuto.

\subsection{Dov'È Oggi IL "Locale" dei Sistemi locali? Contesti e nicChie.}

A questo punto la questione teorica su cui focalizzare l'attenzione appare ben definita. Si tratta di ammettere che lo spazio mondiale dei prodotti ha spessore, 
densità ed irregolarità, e che non è dunque indifferente vivere ed operare in un luogo o nell'altro. Occorre d'altro canto elaborare una raffigurazione concettuale ed analitica che non riduca il "locale" a localismo. Abbiamo esordito sostenendo che il paradigma classico dei SSL non riesce a dare conto congiuntamente dei due versanti. Abbiamo introdotto alcuni strumenti che ci appaiono maggiormente adeguati. Dobbiamo adesso direttamente affrontare la domanda: se oggi il "locale" non coincide con la località, in che cosa consiste?

Una prima risposta, assieme a Michael Storper (2009), prende le mosse da proposizioni largamente accettate tra gli scienziati sociali: l'informazione specializzata rilevante non è uniformemente disponibile, in quanto costosa e limitata da barriere di vario tipo; le nostre decisioni si orientano molto spesso sulla scorta di criteri di riferimento, consuetudini e quadri interpretativi; i network socio-economici in cui siamo immersi regolano in elevata misura il grado e la qualità dell'accesso alle informazioni, nonché la prospettiva di senso con cui ne fruiamo; infine, la posizione relativa all'interno dei network influenza, per emulazione o per differenziazione, il formarsi delle nostre preferenze e finalità. Appoggiandoci a quelle proposizioni possiamo articolare la categoria teorica del "contesto". Essa confronta due ambienti sociali e rileva che se, rispetto all'uno, l'altro altera il frame interpretativo delle alternative accessibili, modifica altresì le modalità della scelta e dell'apprendimento per gli attori.

Possiamo imbatterci in contesti internamente segmentati, ad alta organizzazione e specializzazione, di stampo comunitario, orientati al mercato, e così via. Può altresì mutare la scala geografica del contesto, che talvolta si basa sulla contiguità locale degli attori e talvolta su relazioni a distanza tecnologicamente mediate. Lo snodo teorico per nulla banale che corre sotto tutte queste catalogazioni è che, nel passaggio dall'uno all'altro contesto, la sostituibilità dei tasks (oppure, quando vale ancora l'ottica tradizionale, dei beni finali) è imperfetta, proprio perché il mutamento del contesto cambia la selezione e la combinazione delle alternative e la natura dei processi di apprendimento. Quasi mai possiamo replicare un task o un bene come una fotocopia, in quanto è il contesto che rende parziale il grado di sostituibilità. Mentre in un saggio famoso per molti studiosi dei sistemi locali (Becattini-Rullani, 1993) sono soltanto le diversità locali ad alimentare i vantaggi competitivi, nella più recente riflessione di analisti, tra i quali Storper, il contesto esiste ed esercita i suoi effetti anche quando è sradicato da un preciso luogo, ossia anche quando è (per riprendere un concetto presentato sopra) "apprendimento trialogico". 
Se il contesto così definito mette in discussione la visione incentrata sull'appiattimento del mondo, mentre esso non è necessariamente locale, esistono dimensioni del SSL che rimangono ancorate ad uno specifico territorio? Per rispondere, dobbiamo considerare alcuni percorsi attraverso cui un SSL può oggi avviare l'innovazione socio-tecnica. Per un verso il SSL è immerso in flussi d'informazione e di conoscenza che non hanno barriere. Per un altro, specialmente al fine di coltivare task capabilities sue proprie, il SSL deve progettare e quindi valorizzare le competenze, le motivazioni, la trama delle connessioni in cui è inserito e che può, entro certi limiti, sviluppare all'interno dei propri confini (la "prossimità geografica"). I due versanti vengono congiuntamente spiegati distinguendo interrelati livelli di complessità: quello micro delle nicchie, quello meso dei regimi e quello macro dei socio-technical landscapes (Rip, Kemp, 1998; Geels, 2004; vedi nota 13).

Fig. 1

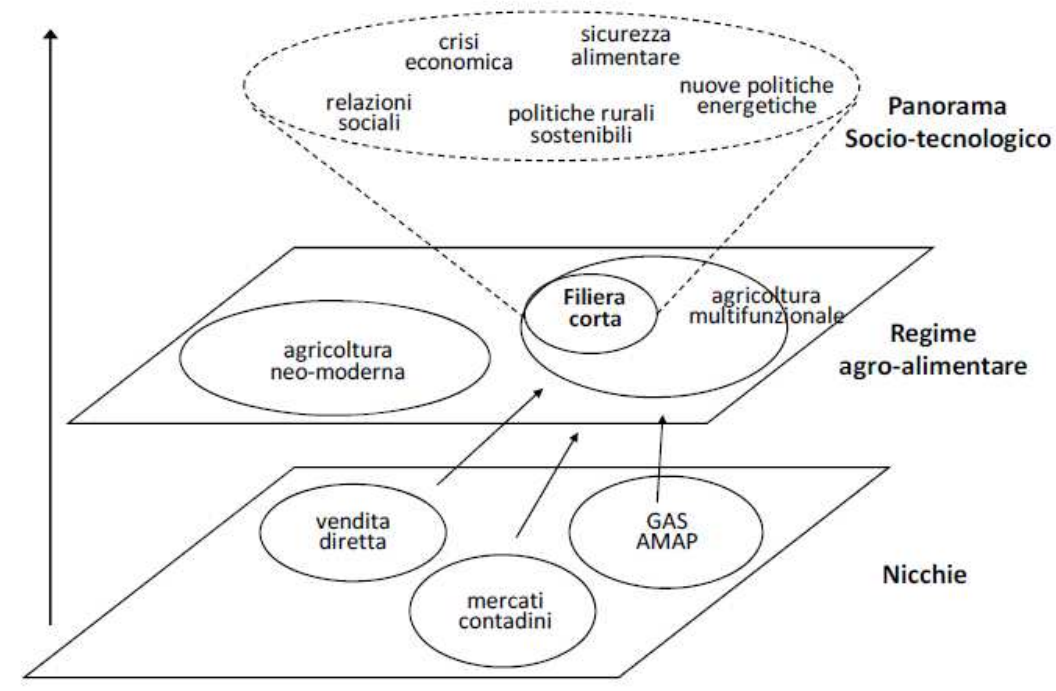

Fonte: Guidi (2009: 62).

Presentiamo (Fig. 1) un particolare adattamento dell'approccio generale, incentrato sull'evoluzione di un sistema socio-tecnico multi-livello (Geels, 2002, 2005), mediante una figura che illustra la transizione di un SSL verso un regime alimentare di filiera corta. Iniziando dall'alto, il "panorama" configura il complesso esistente di istituzioni e mentalità condivise. Il "regime" rappresenta l'insieme di regole socio-tecniche che coordina gli attori e le risorse. Infine, le "nicchie" costituiscono gli ambiti economici protetti in cui si sperimentano processi di produzione, consumo, organizzazione sociale e ricerca. In questo schema i processi innovativi possono essere così definiti: 1) multi-livello, perché i percorsi avviati nelle nicchie possono talvolta trasferirsi al regime, a sua volta modellato da, e al tempo 
stesso capace di influire su, fattori attinenti al livello macro ("landscape"), 2) multidimensionali, in quanto le traiettorie di cambiamento coinvolgono insiemi differenziati di agenti individuali e collettivi che interagiscono: scienziati, tecnici, imprese, consumatori e produttori, enti pubblici,

Uno dei punti più rilevanti della nostra riflessione riguarda il carattere di "apprendimento autogestito" delle nicchie (Bertini, 2007). Queste ultime, che stanno di solito ai margini del regime esistente, affidano ai propri membri la piena responsabilità della soluzione dei problemi e la realizzazione di iniziative mediante la libera scelta di obiettivi, strumenti e organizzazione del lavoro. Tali percorsi sociali creativi non coincidono con quelli delle comunità virtuali del peer to peer o del prosumer. Accanto infatti a motivazioni e competenze spesso simili, I'apprendimento autogestito prevede, a differenza delle comunità virtuali, delle "sessioni di presenza", in cui ci si confronta faccia-a-faccia su significati e valori e si condividono conoscenze di difficile codifica e trasmissione. In breve, stiamo sostenendo che le nicchie sociotecniche sono, negli odierni SSL, gli ambiti in cui rimangono decisive le relazioni di prossimità geografica e d'interazione diretta.

\subsection{SINTESI}

In questa Seconda parte abbiamo argomentato l'esigenza di disancorare la riflessione sui SSL dall'idea fondativa della prossimità geografica. Il nostro ragionamento si è articolato in cinque passaggi concettuali tra loro congruenti. 1] L'idea della task-based production spiega da un lato come stanno cambiando le ragioni per cui le attività economiche si agglutinano in un SSL e dall'altro perché le imprese connotate quali produttrici di determinati beni finali possano non essere più gli attori centrali. 2] L'idea che la de-verticalizzazione delle imprese conduca ad un intreccio inestricabile di contratti formali e accordi informali, entro consorzi capaci di alzare i costi di transizione per trattenere i propri membri, aiuta a comprendere la novità delle collaborazioni inter-imprese. 3] L'idea di una costitutiva ambivalenza dello spazio mondiale dei prodotti enfatizza la propensione a creare gruppi di prodotti "correlati" in base alla prossimità cognitiva, mentre alla capacità di sviluppare ed utilizzare i legami deboli è dovuta la possibilità per un SSL di cambiare anche bruscamente la propria traiettoria. 4] L'idea di "contesto" contribuisce a chiarire perché il mondo non è piatto, grazie alla prospettiva che non rende necessario "situare" il contesto stesso in un ambito territoriale circoscritto. 5] Infine, l'idea dei 
sistemi socio-tecnici contribuisce in ogni caso a conferire un ruolo cruciale al territorio e agli addensamenti di capabilities dinamiche al suo interno: esso rimane importante soprattutto come ambito di intersezione dei flussi multi-dimensionali e multi-scalari di conoscenze, che costituiscono la fonte primaria per la generazione, l'incremento diversificato e lo sviluppo di nicchie innovative.

Tutto ciò specie nei processi di transizione socio-tecnica come quello odierno.

\section{Terza Parte. AlLa RiCerca delle traiettorie inNOVATIVe ALL'INTERNO di UN PROCESSO DI TRANSIZIONE SOCIO-TECNICA}

La serie di processi alla base della nuova divisione internazionale del lavoro a livello globale inducono un cambiamento dello spazio delle produzioni, inteso come evoluzione delle coordinate tecnico-economiche e quindi delle modalità di svolgimento delle fasi. Muta la configurazione dei cicli produttivi, con attività e funzioni che assumono una rilevanza trasversale tra più settori, molti dei quali sono dispersi in territori talvolta molto lontani. Ciò implica che gli spazi per Paesi, imprese e aree si trasformano incessantemente sulla base di una dinamica innovativa accelerata ${ }^{24}$. In questo scenario diviene essenziale l'attività di raccolta e sistematizzazione delle informazioni, al fine di delineare macro-traiettorie rispetto alle quali individuare ambiti di azione e opportunità.

Tutto ciò acquista particolare importanza nel contesto odierno, che contiene un mix apparentemente imprevedibile di processi involutivi e potenzialità che si profilano. Non si tratta di fare esercizi di futurologia, né di discutere previsioni ottenute mediante estrapolazioni da trend rilevati per il recente passato. Occorre in realtà sviluppare tentativi volti ad individuare mutamenti tali da generare possibili effetti di grande importanza. In altri termini, occorre definire delle coordinate generali partendo da punti ben acquisiti, ovvero dall'individuazione di eventi e processi che possiamo considerare irreversibili e quindi da assumere come direttrici di orientamento. Svilupperemo a tal fine la riflessione basandoci soprattutto su studi strategici elaborati in Germania nell'ultimo decennio (Deutsche Bank Research, DBR, 2007, 2008, 2009a,b).

\footnotetext{
${ }^{24}$ Per un'analisi della dinamica evolutiva degli "spazi di produzione" per aree continentali e Paesi dal 1996 al 2004 si veda Lombardi et al (2009), dove viene applicata una particolare metodologia, basata su stime dell'evoluzione del RCA attraverso un algoritmo di valutazione della "prossimità settoriale" all'interno dei vari Paesi.
} 
La crisi odierna accelererà il ritmo del cambiamento strutturale in atto da tempo, ovvero l'evoluzione della composizione settoriale e della struttura occupazionale, nonostante il declino del commercio mondiale abbia provocato forti contrazioni del PIL (-6\% nel 2009), della domanda di beni d'investimento proveniente da molte economie mondiali e del livello di utilizzazione degli impianti (al $70 \%$ anche dopo i recenti impulsi positivi). La dinamica strutturale non sarà uniforme, ma avrà impatti differenziati sui settori e sulle economie, a seconda della modalità con cui si combineranno con i macro-trend internazionali.

Pur nella sua gravità, tutto questo rallenta, ma non blocca, la nuova divisione del lavoro a livello internazionale, data l'azione congiunta di alcuni drivers del processo di globalizzazione: 1) sviluppi nel campo delle tecnologie dell'informazione, 2) riduzioni nei costi di trasporto, 3) cambiamenti climatici e prezzi degli input energetici, 5) cambiamenti tecnologici nelle infrastrutture di comunicazione e nella logistica a livello mondiale.

Su queste basi tenderà a consolidarsi la dinamica di un "mondo multipolare", contraddistinto da una pluralità di "motori della crescita", anche se gli USA rimarranno il principale mercato nella prossima decade. Nel decennio in corso le emerging economies hanno registrato i tassi di crescita più elevati, da cui è derivato un incremento della loro quota sulle esportazioni mondiali dal $28,1 \%$ al $31,4 \%$. Nel prossimo cambierà lo spazio geo-economico, nella misura in cui sarà modificata la struttura degli impulsi provenienti dalle varie regioni: alcune di queste continueranno il percorso dinamico intrapreso e quindi incrementeranno il ritmo di espansione di una domanda globale di beni profondamente modificata nella sua struttura. In questo scenario è destinato ad aumentare il fabbisogno delle economie emergenti di un insieme vasto e composito di beni essenziali per innescare e rafforzare i processi di crescita: meccanica strumentale di contenuto medio-alto, attrezzature per le dotazioni infrastrutturali, prodotti e tecnologie innovative per la produzione e il consumo efficiente dell'energia, beni di consumo di "gamma alta". Si tratta, come si vede, dello scenario imperniato su un enorme sviluppo degli scambi a livello mondiale, dove è cruciale la capacità di individuare spazi di offerta produttiva, in modo che possano combinarsi efficacemente con la domanda rilevante da un punto di vista quantitativo e molto interessante sul piano qualitativo, perché implica ulteriore sviluppo di nuove tecnologie.

La Germania è stata finora in grado di intercettare una parte cospicua di tali flussi fino a raggiungere e mantenere posizione di leader globale delle esportazioni di 
merci, prima della Cina e degli Usa. Le tendenze delineate non sorgono improvvisamente, ma sono iscritte in un'evoluzione da lungo in atto e individuata con precisione sia in Germania (DBR, 2007) che negli Stati Uniti (Rocky Mountains Institute, 2004).

Due sono i macro-trend globali con cui si è aperto il secolo attuale e che sono destinati a caratterizzarlo nei prossimi decenni:

I. Fine dell'era del petrolio ("the end of oil era").

II. Creazione di nuove infrastrutture materiali e immateriali, insieme all'ammodernamento di quelle esistenti verso finalità indicate nei punti successivi.

Dal punto $\mathrm{I}^{\circ}$ discendono una serie di imperativi/direttrici di lungo periodo per le economie di tutto il mondo:

1) Aumento esponenziale della domanda mondiale di input energetici.

2) Riduzione intensa dei fabbisogni e dei costi degli stessi.

3) Squilibri tra domanda e offerta e quindi necessità di diversificare le fonti e al tempo stesso rendere molto più efficiente il loro uso.

4) Ri-modellamento dei processi produttivi praticamente di tutti i beni. Si pensi all'industria dell'automobile, che deve rispondere a sfide del tutto nuove ed è soggetta a innovazioni tecnico-scientifiche di enorme portata.

Dalla creazione di nuove infrastrutture (punto $\mathrm{II}^{\circ}$ ) derivano alcune traiettorie di espansione per un insieme complesso di attività e funzioni:

1) Installazione di attrezzature e strutture di differente natura e a molteplici scale geografiche, in seguito all'ulteriore sviluppo di dispositivi di interconnessione al tempo stesso ramificati gerarchicamente ed efficienti. Su tali strutture devono circolare flussi crescenti di merci e informazioni.

2) Sviluppo di infrastrutture immateriali, quali dispositivi distribuiti e centralizzati per l'elaborazione delle informazioni, in modo da consentire la preservazione delle strutture e l'organizzazione dei flussi.

È agevole comprendere che si tratta di un enorme potenziale di sviluppo, che racchiude una serie di ambiti e spazi produttivi a vari livelli. Lo scenario diviene ancora più rilevante se si pensa all'evoluzione quantitativa e qualitativa della domanda di beni di consumo connessa al consolidamento dello sviluppo delle emerging economies. È anzi fondamentale assumere la centralità dello stretto connubio dinamico tra tutti gli elementi in gioco. Ciò costituisce un buon esempio di come un Paese e alcune aree economico-produttive possono ricollocarsi in uno spazio mondiale di prodotti in 
continua evoluzione, sulla base di potenzialità per il sistema opportunamente individuate. Ebbene, i macro-trend descritti e le connesse traiettorie hanno costituito il quadro generale di riferimento, entro il quale è stato concepito il ruolo e la funzione dell'"ingegneria meccanica", ovvero di quella parte dell'industria meccanica in grado sia di fornire impianti, beni strumentali, attrezzature, meccanismi per "macchine produttive" in via di formazione", sia di offrire strumenti e servizi per la crescente domanda mondiale di beni e funzioni collegate all'impiego di input energetici. In questa prospettiva il fondamento del potenziale produttivo tedesco e dei suoi successi risiede nell'industria meccanica ad alta intensità di conoscenze, dal momento che I'offerta di macchine, attrezzature e impianti incorporano necessariamente dispositivi in grado di rispondere alle complesse esigenze precedentemente indicate. Al tempo stesso, però, ciò non ha impedito l'espansione di funzioni terziarie, anzi è stato proprio quella traiettoria a richiedere una dotazione terziaria, che è peraltro ritenuta al di sotto delle necessità inerenti al potenziale produttivo dispiegato. Quelle appena descritte sono le modalità con cui la Germania è entrata nella "società della conoscenza", di cui ha colto immediatamente la portata globale e lo spazio di possibilità per il sistema economico nel suo insieme, a partire da un nucleo propulsore.

In breve, dall'esperienza tedesca emergono con chiarezza tre punti basilari:

1. Le strategie di orientamento del potenziale produttivo devono commisurarsi con i risultati di ricognizioni sistematiche dello spazio economico globale e delle potenzialità da esso generate.

2. I processi di trasformazione efficaci nell'odierno scenario richiedono combinazioni industria-terziario tali da richiedere funzioni elevate di natura trasversale per settori e attività. Di conseguenza è sempre più confermata la tesi che le tradizionali tipologie classificatorie sono obsolete.

3. L'adozione di una prospettiva dinamica ed evolutiva nell'analisi e nell'elaborazione di strategie è essenziale, data l'intensità e l'estensione della dinamica in atto.

Alla luce dei punti enucleati appare fondata la tesi che siamo entrati in una fase di transizione socio-tecnica, nel corso della quale si verificheranno mutamenti significativi a vari livelli: nuove nicchie, nuovi regimi tecnologici, alterazioni del tecnological landascape. In tale orizzonte devono essere analizzate e discusse le trasformazioni in atto nella divisione internazionale del lavoro, le chances per l'Europa e per i Paesi europei, con i SSL al loro interno Da ciò discendono poi le prospettive di 
crescita di imprese, regioni e paesi, come anche i risultati più o meno brillanti conseguiti in questi anni. Le economie più sviluppate devono misurarsi con questi aspetti fondamentali traendone implicazioni in termini della necessità di riflettere sistematicamente sul proprio posizionamento strategico all'interno di una dinamica strutturale accelerata.

Appare altresì evidente che i SSL costituiscono elementi essenziali del potenziale di sviluppo di un Paese e di un sistema economico-produttivo posto di fronte alle sfide indotte dal processo di transizione.

\section{QuARTA PARTe. QUALe POLICY È OGgI PeRCoRRIBILe?}

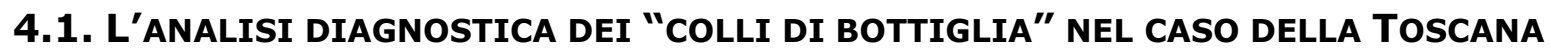

Vale per I'intera gamma dei SSL ciò che lucidamente Viesti (2000: 171) sintetizza per i distretti: «Strategie che mirino a trapiantare altrove distretti di successo o a pianificare l'imitazione, in altri luoghi, di distretti esistenti, sono destinate nella maggior parte dei casi al fallimento. Questo però non significa affatto che le politiche di sviluppo siano irrilevanti. Tutt'altro. Esse sono importanti nel fornire alle imprese le risorse che sono il prerequisito dello sviluppo di ogni area: legalità, istruzione, infrastrutture di base; e via via beni pubblici più rari e sofisticati, da università di qualità a infrastrutture avanzate di trasporto e comunicazione. Poi, sta in molti casi alle politiche di sviluppo realizzare le istituzioni che possono accompagnare lo sviluppo delle imprese: un sistema finanziario in grado di selezionare gli impieghi e di investire in loco il risparmio disponibile (o di attrarre risparmio) è condizione fondamentale; un sistema dell'istruzione e della formazione professionale è cruciale: una forza lavoro istruita e formata professionalmente è il fattore in assoluto più importante. Sono utili politiche che favoriscono il mantenimento, il rafforzamento, l'aggiornamento delle culture locali, e allo stesso tempo è cruciale la massima apertura verso apporti esterni, l'attrazione di investimenti, di imprenditori, di capitale umano qualificato».

È dunque chiaro, e tante volte ribadito, che cosa la policy dovrebbe realizzare. Ciò non sposta di un millimetro i termini del problema teorico-politico che qui ci poniamo: che cosa possiamo ottenere dalla policy, quando essa è, nell'ambito di un SSL, paralizzata da veti incrociati, influenzata da rendite parassitarie, incapace di 
dialogare con chi ha idee e risorse? L'inadeguatezza della policy effettiva rispetto a quella "dottrinaria" appare ancora più elevata alla luce della diagnosi proposta nelle pagine precedenti, secondo cui anche la Toscana ha l'esigenza di una discontinuità di sistema che, mediante azioni concertate di provenienza pubblica, civile e privata, allestisca «strategie su una molteplicità di dimensioni (tecnico-scientifica, economicofinanziaria, culturale, formativa, normativa) e sviluppi una progettualità aperta, che sappia coniugare elaborazione delle conoscenze e scelta delle linee di azioni migliori in condizioni di incertezza e complessità crescenti» (IRPET, 2009: 255).

Che fare, dunque? Il recente dibattito italiano sugli interventi di policy per lo sviluppo locale ha espresso, ci sembra, due posizioni polari. Ad un estremo, è stata propugnata l'istituzione di «un'agenzia a competenza sovra regionale, dotata di risorse sufficienti (da attingere anche a quelle comunitarie [...]), che agisca in "isolamento" operativo e discrezionalmente, sulla base di scelte strategiche generali circa le aree territoriali, le innovazioni e la specializzazione produttiva da promuovere o rafforzare. La formula da perseguire è quella di consentire la localizzazione "chiavi in mano" di attività fortemente innovative, ad alto valore aggiunto e orientate all'export, possibilmente gestite da società già caratterizzate da un curriculum di successo, meglio se straniere» (La Spina, 2003: 366). All'altro estremo, si è puntato ad azioni strategiche negoziali e partecipative che, nel perimetro di un SSL, convincano «molti liberi attori diversi, di diversi ambiti funzionali e istituzionali, a comportamenti che si orientano reciprocamente, ovvero a investimenti complementari lungo un sentiero di crescita definito da scelte sulle quali si raggiunge un ragionevole consenso e sui cui esiti si possono fare ragionevoli previsioni» (Bagnasco, 2003: 116). L'una suggerisce una struttura apolitica e tecnocratica paracadutata dal cielo e difesa in un bunker. L'altra esalta le capacità auto-organizzative delle società locali, intese tanto come società civili quanto come società politiche. La prima posizione appare debole in quanto un territorio rigetta i bunker non già espugnandoli, bensì proprio riaffermando I'isolamento con cui sono stati progettati, ossia lasciandoli implodere per carenza di informazioni e risorse dall'esterno. La seconda alimenta un nobile wishful thinking, perché lo scarso spessore di tanti segmenti dell'odierna società civile e di quasi l'intera società politica sono un "duro fatto" che non si aggira con collaborazioni multilaterali negoziate con stile pragmatico e incrementale.

È individuabile un differente frame di analisi teorica e progettazione operativa che, pur tenendo in serissimo conto i limiti delle società locali nostrane, eviti d'indossare l'elmetto e il bazooka del tecnocrate? Alcuni elementi di risposta, entro il 
nostro campo disciplinare, vengono dalla cosiddetta "economia clinica", secondo cui il compito e la possibilità dello scienziato sociale sta nell'interpretare complessi di sintomi, mai causalmente univoci e unidirezionali, per elaborare una sindrome e proporre una terapia nella quale, come accade quando il medico prescrive farmaci o interviene chirurgicamente, i criteri siano di concentrarsi sulla patologia principale e di arrecare benefici diretti superiori ai danni indiretti ${ }^{25}$. Vi sono esperienze reali di processi di transizione socio-tecnica, progettati e attuati, sia pure non senza difficoltà e problemi, in altri paesi (Rip e Kemp, 1998; Kemp, Schot e Hoogma, 1998; Geels e Schot, 2007, Frenken et al., 2003; Hekkert e Negro, 2009). Da esse traiamo spunti di riflessione nell'esplicitare il nostro approccio fino al livello operativo.

Questo approccio muove da alcune valutazioni preliminari. Innanzitutto, se accettiamo I'idea diffusa che I'apparato economico-produttivo toscano stia incontrando difficoltà crescenti nel fronteggiare la dinamica innovativa odierna, può apparire fondata un'impostazione fondata su un "sano gradualismo". Un'idea consolidata è infatti che, per favorire il cambiamento dove è assente o debole, occorrono varie riforme economiche sulle quali, in astratto, il consenso tra gli esperti è ampio. Poiché un approccio di first best, che eliminerebbe d'un colpo tutte le manchevolezze e distorsioni, sta nel libro dei sogni e non si commisura ai processi reali, prevale la concezione secondo cui do as much reform as you can, as best as you can, in quanto (a) ogni riforma è buona; (b) "più aree si riformano, meglio è; (c) più profonda è la riforma in un'area, meglio è" (Rodrik, 2008). Si tratta però di una visione sempre meno in grado di gestire sistemi con gradi variabili di complessità e incertezza, in relazione alle traiettorie tecno-economiche in cui sono inseriti. Nell'impostazione che proponiamo un criterio ispiratore è quello di uno scenario caratterizzato da una catena provvista di parecchi "anelli deboli", ma non sappiamo quali e quanti essi siano, né il loro preciso livello di debolezza. In tali circostanze rafforzarne uno - mediante una riforma parziale - può essere irrilevante, o addirittura può peggiorare la situazione, se quel rafforzamento accresce la "tensione" sugli anelli vicini, e tra questi ve ne sono altri molto deboli. In termini teorici, è questo che ha dimostrato, oltre cinquant'anni $\mathrm{fa}$, il fondamentale teorema del second best ${ }^{26}$.

\footnotetext{
${ }^{25}$ In ambito di Economia dello sviluppo, procedono lungo linee metodologiche simili autori che pure, sotto il profilo teorico, sono tra loro distanti: si vedano in particolare Sachs (2005, cap.IV) e Hausmann et al. (2008).

${ }^{26}$ Questo teorema dimostra che una riforma di per sé, in presenza di altre distorsioni, non assicura la promozione del benessere. Una situazione in cui un vincolo impedisce di ottenere anche una sola delle condizioni di ottimale funzionamento dei mercati (la cosiddetta "efficienza paretiana"), comporta che le altre condizioni, pur essendo ancora raggiungibili, non siano più in generale desiderabili. Se cioè in un sistema economico sono presenti deviazioni dal modello ideale, un intervento di policy che miri a correggere qualcuna di tali distorsioni non assicura una maggiore efficienza al sistema nel suo complesso,
} 
La prospettiva qui suggerita muove da alcuni elementi fondamentali, connessi all'analisi sviluppata nei paragrafi precedenti e che brevemente richiamiamo.

Nell'odierna fase di evoluzione tecno-economica a livello internazionale i SSL sono inseriti in sistemi socio-tecnici, la cui dinamica è complessa e imprevedibile, in conseguenza di processi multi-dimensionali e multi-scalari. La centralità assunta dalle dinamiche reticolari e quindi dalla varietà morfologica degli stessi network genera un'ampia gamma di effetti, di analizziamo alcune tipologie, data la loro fecondità analitica per comprendere processi evolutivi e talvolta involutivi, che hanno caratterizzato gli stessi SSL. Innanzitutto la dinamica economica si basa sulla coevoluzione di tecnologie, modelli organizzativi, culture, istituzioni; da ciò discende la presenza di una molteplicità di traiettorie possibili, tra le quali processi selettivi complessi portano all'emergere di uno o più sentieri di sviluppo, grazie all'azione di determinati meccanismi, ben analizzati in numerosi studi (Arthur, 1994; Foxon, 2006; Unruh, 2002; Unruh e Hermosilla, 2006, per citarne solo alcuni): economie di scala e di apprendimento, meccanismi generativi e aspettative adattative, economie di rete, accumulazione di know how al tempo stesso complesso e specifico. Su queste basi e a determinate condizioni si realizzano "combinazioni di forze sistemiche" in grado di produrre "circuiti virtuosi" auto-rinforzantisi, con rendimenti crescenti causati dall'adozione di precise direttrici tecnologiche. Emergono così paradigmi tecnoeconomici e corrispondenti modelli socio-organizzativi, al cui interno si sviluppano reti interattive. Il fatto è che l'insieme di fattori descritti possono assumere una duplice valenza: da un lato di innesco e rafforzamento di processi espansivi, dall'altro di fattori di "blocco" (processi di lock-in), tali da indurre barriere rispetto a dinamiche di mutamento ed elementi di vulnerabilità strutturale nel lungo periodo. Può accadere che un sistema socio-tecnico e i SSL ad essi correlati, in grado di prosperare in un dato orizzonte tecno-economico, non riescano nemmeno a percepire i segnali delle dinamiche di trasformazione connesse a processi multi-dimensionali. I circuiti virtuosi si trasformano così in "circoli viziosi" e in processi di implosione sistemica. Fenomeni di questa natura investono in modo particolare i SSL qualora le "interazioni forti"

\footnotetext{
poiché introduce esso stesso un'altra distorsione (quali, ad esempio, imposte, sussidi, regolamenti, e così via). Pertanto l'allontanamento da una condizione necessaria per l'efficienza richiede l'allontanamento da tutte le altre condizioni necessarie in un senso non precisabile in generale. Inoltre in parecchi casi nemmeno conosciamo l'intera lista delle distorsioni (ad esempio, quelle derivanti da fallimenti dei mercati o da imperfetta credibilità), né possiamo selezionare le riforme che enfatizzano (almeno) le interazioni positive di second best, poiché gran parte di tali effetti possono difficilmente essere colti e misurati ex ante. In breve: il "mondo ideale" dell'economia efficiente è, ed è destinato a rimanere, un ideale platonico. Sappiamo in astratto quali ingredienti lo compongono. Ma basta che ne manchi qualcuno per "smarrire la strada": non siamo più in grado di capire se una "riforma parziale" ci sta avvicinando o meno all'ideale.
} 
prevalgano nettamente su quelle deboli fino a farle diventare debolissime, causando di conseguenza l'emergere di percorsi di auto-referenzialità e di prevalenza di culture, tecnologie e modelli istituzionali adatti ad un technological landscape ormai superato.

La questione fondamentale che si pone per molti SSL è allora la seguente: come misurarsi con le sfide indotte da una fase di transizione socio-tecnica come quella odierna? La riflessione sul piano strategico operativo non può essere svincolata dalle acquisizioni dell'analisi svolta, soprattutto in relazione al fatto che occorre pensare linee strategiche per dinamiche incentrate su strutture interattive tra molti agenti a differente scala.

\subsection{TOSCANA: CHE FARE, QUI ED ORA?}

\subsection{1 "Non esiste la PANACEA" (OStrom et AL, 2007)}

Se dunque modificare "secondo i dettami degli esperti" questo o quel pezzo dei SSL non ci garantisce di stare procedendo verso il meglio, la Scienza deve coniugarsi con I'Arte, intesa come interpretazione diagnostica dei sintomi e abilità nel cogliere pochi rimedi praticabili. Sta qui la scommessa teorico-politica che ci appassiona, partendo dalla consapevolezza propria di un numero crescente di studiosi ${ }^{27}$. Occorre superare approcci basati sull'idea di proposte univoche e risolutive (going beyond panaceas) e partire da un approccio diagnostico, che abbia al centro il riconoscimento dei sistemi complessi, con precise caratteristiche: non linearità, dipendenza da molte variabili a differente scala, natura evolutiva, multi-scalarità dei processi di cambiamento. Non possono esistere rimedi cosiddetti once for all, specialmente durante le transizioni socio-tecniche, allorquando è necessario prendere in considerazione molti processi e variabili, unitamente al ruolo fondamentale di una molteplicità di soggetti, peraltro all'interno di apparati economico-produttivi alle prese con fenomeni di lock-in cognitivo, organizzativo, tecnico-scientifico.

Il punto di partenza di una riflessione appropriata non può allora che essere la seguente assunzione: nel corso delle fasi di transizione esiste un'aporia di fondo tra interesse di breve periodo (sopravvivenza dell'esistente) e interesse di medio-lungo termine (innesco di una o più nuove traiettorie). Su tale aporia si innestano aggregazioni di interessi e conseguenti strategie degli agenti che ne sono portatori; di qui deriva poi la differenziazione tra le linee di sviluppo di un sistema socio-economico e gli orizzonti differenziati degli attori, magari inseriti in strutture connettive

27 Si veda Ostrom et al (2007) e la letteratura di riferimento ivi citata, che si riferisce ad un ampio insieme di studi in molti campi (tecnico-scientifico, economico-sociale, ecologico, culturale) 
tendenzialmente globali. E' elevato il rischio di processi involutivi, conseguenti a interazioni e feedback tendenti solo a perpetuare l'esistente, con probabili esiti di system failure. $E^{\prime}$ al tempo stesso ampio, però, il campo di potenzialità che si apre, il cui innesco dipende dalla capacità di avviare feedback positivi in nuove direzioni, che sappiano coniugare orizzonti e interessi di lungo termine con nuove istanze di breve periodo. Nella prospettiva delle transizioni socio-tecniche vi sono basilari imperativi metodologico-concettuali, a cui sarebbe opportuno ispirarsi ${ }^{28}: 1$ ) ampliare lo spettro di strategie connesse, grazie a progetti mirati di innovazione multi-dimensionale, 2) promuovere e sostenere differenziazioni strategiche, in modo da favorire flessibilità strutturale e adattativa, 3) perseguire una gamma di opzioni per investimenti strategici, 4) stimolare l'azione di agenti innovatori ("propulsivi") ed ipotizzare traiettorie tecnologiche rilevanti, 5) creare le condizioni per la diffusione di strutture interattive a varia scala.

Tali imperativi possono trovare direttrici operative mirate sulla nostra realtà di riferimento (Toscana), per la quale si pone in primo luogo l'esigenza di rimuovere fattori di blocco oppure ostativi verso processi di mutamento strutturale.

\subsubsection{LINEE STRATEGICO-OPERATIVE PER LA TOSCANA: SPUNTI INTRODUTTIVI}

Dobbiamo elaborare ipotesi sui "colli di bottiglia" (bottlenecks, fattori di lock-in) del particolare SSL in esame o di una regione vista come insieme di SSL, per selezionare poche priorità d'intervento e un numero appropriato di mosse realistiche. Siccome siamo in un periodo di transizione socio-tecnica, la nostra ipotesi, esplicitamente unilaterale ma non avulsa dai fattori causali della debolezza strutturale $^{29}$, è che la Toscana possa misurarsi con le sfide emergenti affrontando tre principali "colli di bottiglia" che indeboliscono sostanzialmente il suo odierno potenziale di sviluppo: uno è cognitivo e riguarda la miopia nei riguardi dei macro-trend socioeconomici sopra richiamati e delle potenzialità che in essi si annidano; un altro sta nell'inadeguatezza delle forme organizzative delle attività economiche; l'ultimo è rappresentato dalla carenza di appropriate formule istituzionali nell'ambito della

\footnotetext{
${ }^{28}$ Si tratta di una riformulazione di punti trattati in una vasta letteratura. Riferimento essenziale ai nostri fini è Rammel e Van den Bergh (2003).

${ }^{29}$ Le criticità dei SSL toscani in termini dimensionali e di specializzazione produttiva sono analizzate in molti lavori di economia applicata; le rigidità ed inefficienze del mercato del lavoro; la mancanza di un sistema infrastrutturale moderno; la vastità delle aree di rendita; il progressivo invecchiamento della popolazione; la difficile condizione della finanza pubblica; e così via. Con l'espressione "colli di bottiglia" vengono in realtà sintetizzati una serie di elementi critici, agendo sui quali è possibile innescare un "effetto leva" per avviare feedback positivi e quindi proficui mutamenti socio-tecnici di lungo periodo.
} 
capitalizzazione delle imprese innovative. La nostra riflessione sarà necessariamente stringata e diretta principalmente a mettere in risalto i punti ritenuti decisivi.

Gli imperativi prima enunciati implicano un ruolo fondamentale per le istituzioni pubbliche locali, che dovrebbero intervenire in funzione delle esigenze innovative delle imprese di un SSL o di un insieme di $S_{S L}{ }^{30}$. Poiché i processi di transizione sociotecnica sono in realtà complessi e attengono a molte dimensioni, i lock-in possono essere superati solo generando impulsi e mettendo in moto meccanismi tali da indurre dinamiche interattive tra agenti socio-economici in nuovi spazi tecnico-produttivi. L'obiettivo è ovviamente non semplice, ma è nondimeno essenziale perseguirlo per evitare rischi di marginalità e di implosione sistemica.

In un siffatto orizzonte, dati i tre "colli di bottiglia" ipotizzati, suggeriamo di concentrarsi su tre livelli: informativo, organizzativo e di start-up di imprese innovative.

Livello: informativo. Centralità di nuovi spazi ontologici.

Partiamo dalle esigenze dell'apparato produttivo. Da una parte, occorre che l'imprenditore riesca davvero a dialogare con le istituzioni locali. Dall'altro, queste ultime debbono orientare l'imprenditore rispetto ai macro-trend evolutivi dell'economia globale.

In aree economico-territoriali come la Toscana soprattutto le piccole imprese si trovano di fronte a problemi che possono essere così sintetizzati: 1) Sorgono esigenze di introdurre varianti di prodotto o di processo, ma le PI non posseggono le conoscenze adeguate per risolvere i problemi. Procedono quindi per adattamenti, basati sull'apprendimento empirico/esperenziale, cioè provando mutevoli ipotesi di lavoro, che modificano fino a trovare qualche soluzione parziale e temporanea. 2)

\footnotetext{
30 Manca lo spazio per una disamina minimamente adeguata degli enti pubblici. Riportiamo tuttavia un brano di Scarlato $(2010 ; 16)$, che esprime una linea di policy coerente con il complesso delle nostre riflessioni: I'autrice prende spunta da una recente innovazione nel modello di governance territoriale, che è in discussione nella Regione Campania, e che è denominata "accordi di reciprocità", cioè accordi che sottendono relazioni in cui tutte le parti assumono responsabilità reciproche. Tali accordi dovranno essere siglati tra la Regione e un Ente intermedio, formato dall'aggregazione di più Comuni, che presentino un progetto su come realizzare gli Obiettivi di servizio e altri target strategici. La novità è che questi accordi consentono un decentramento di responsabilità ai Comuni con relativa assegnazione di risorse, predisponendo strumenti di selezione sulla base di criteri come la qualità del progetto, la concentrazione della spesa, l'ampiezza del partenariato locale. Tutti elementi che dovrebbero permettere un superamento dei limiti presenti negli strumenti di programmazione negoziata utilizzati in passato (cosa dire della vaghezza del concetto di "idea forza" come elemento distintivo dei vecchi progetti?). In questa cornice istituzionale rinnovata, potranno essere inseriti interventi su più fronti (riqualificazione urbana, servizi collettivi, ecc.) che coinvolgono potenzialmente l'imprenditorialità locale, profit e non profit. Ma va sottolineato che le risorse sono destinate per il $70 \%$ al finanziamento di infrastrutture materiali e immateriali. È stata quindi capovolta la logica dei Patti territoriali, che hanno prevalentemente fornito incentivi alle imprese e alimentato in questo modo comportamenti opportunistici».
} 
Esse si trovano di fronte a questioni che non sanno risolvere, pur avendo vaga consapevolezza che l'obiettivo non sarebbe irraggiungibile. 3) I fabbisogni latenti si esprimono in insoddisfazioni $e$ in sensazioni di poter fare meglio, senza però intravedere linee di uscita dall'impasse. 4) Non viene nemmeno previsto l'insorgere di alcuni problemi, dal momento che le imprese in questione sono ripiegate su orizzonti operativi troppo ravvicinati e non hanno la strumentazione tecnico-scientifica per monitorare l'orizzonte tecnologico. 5) Non di rado le esigenze possono presentarsi in modo confuso e ambiguo, favorendo così il ritardo di percezione e quindi la mancata consapevolezza.

A fronte di un sistema produttivo con le descritte proprietà vi è un potenziale tecnico-scientifico di rilevante consistenza, fatto di competenze e linee di ricerca con immediate possibili ricadute per l'apparato produttivo. Per non parlare della possibilità di accedere a spazi tecnico-produttivi grazie a strutture di connessione a scala ampia. Tutto ciò è precluso dal divario tra competenze possedute, tipiche del tradizionale regime tecnologico, e quelle richieste dalle nuove traiettorie tecno-economiche. In altri termini, le imprese sono di fronte alla necessità di ridefinire le ontologie di progettazione e di intervento.

Cosa significa? L'idea di fondo è quella di creare uno spazio delle conoscenze organizzato, inteso come dominio conoscitivo strutturato e combinatoriale, che abbia le seguenti proprietà:

I. Carattere open e friendly delle modalità di accesso, in maniera tale che qualunque agente economico con propensioni innovative, pur senza adeguata preparazione tecnico-scientifica, possa porre le domande nelle formulazioni che ritiene più opportune. Queste ultime devono poi essere trasformate secondo modalità in grado di sviluppare correlazioni con l'insieme degli agenti tecnicoscientifici.

II. Auto-organizzazione dei flussi di conoscenze, nel senso che deve essere possibile lo sviluppo di processi random e bottom-up dalle esigenze alle competenze e all'inverso; per questa via possono autonomamente crearsi le condizioni per modalità interattive finalizzate all'identificazione dei problemi e alla loro soluzione.

III. Dynamic matching tra conoscenze teoriche e/o applicate da un lato e conoscenze esperenziali (on site) dall'altro, attraverso lo sviluppo di un mix di A) impulsi endogeni, che possano liberamente esprimersi e cercare autonomamente le possibili competenze con cui interagire per la risoluzione di problemi; B) impulsi 
esogeni, generati da chi organizza l'open space secondo lineamenti strategici enunciati chiaramente.

IV. Predisposizione di una serie di meccanismi di propulsione/focalizzazione, i quali preparino il terreno e in qualche modo rafforzino tendenze spontanee all'autoorganizzazione dei flussi. Si vedano i precedenti punti IIIA e IIIB, da realizzare mediante progetti congiunti mirati Università-industria e la costruzione di team di competenze multiple (economisti, ingegneri, tecnologi, esperti).

Ciò significa che questo dominio possiede un'articolazione logica in grado di leggere potenzialmente qualsiasi tipo di input (richiesta, esigenza, problema) e interpretarlo alla luce del quadro che organizza lo spazio delle conoscenze. Parte integrante di quest'ultimo è la costruzione di un sotto-spazio delle competenze, ovvero dell'insieme organizzato -su basi conoscitive e tassonomiche- delle competenze esistenti presso le Università e i centri di ricerca.

L'ipotesi quindi si basa sulla creazione di un open space, ma con l'avvertenza che non siamo in presenza di uno spazio totalmente definito, bensì di un assetto basato su regole tali da consentire di individuare nuove connessioni ed eventualmente sviluppare nuove combinazioni (natura combinatoriale). In altri termini, siamo di fronte ad uno spazio conoscitivo organizzato, al cui interno occorre predisporre "macchine" in grado di sviluppare associazioni sistematiche tra il sottospazio delle domande (reinterpretate) e quello delle competenze.

L'ulteriore sviluppo delle connessioni dipende poi dall'interazione diretta degli agenti.

In questa prospettiva non si tratta di predisporre uno swithcboard che smista gli impulsi, bensì di un vero e proprio sistema conoscitivo che evolve realizzando combinazioni grazie ad un "motore ontologico". ${ }^{31}$

Questa linea strategico-operativa può essere realizzata solo se si attua una stretta correlazione strategica tra soggetti (Università, Istituzioni locali, Associazioni di imprese, Banche), in un quadro di convergenza su obiettivi tecno-economici di lungo termine ben definiti. A questo proposito la gamma delle opzioni possibili, sulle quali indirizzare sforzi mirati, è enorme: 1) realizzare quelli che sono definiti intelligent transport systems (ITS) ${ }^{32}$; in breve integrazione tra differenti sistemi di trasporto

\footnotetext{
31 Questa ipotesi progettuale non ha natura solo teorica, in quanto è già un progetto elaborato dall'interazione di quattro Dipartimenti dell'Università di Firenze, oltre al Centro Servizi della stessa Università. Essa si ispira ai principi del broadcast system realizzato negli USA, ma con alcune varianti sostanziali, tra cui appunto il tentativo di creare un motore ontologico.

32 Gli ITS sono al centro di studi statunitensi e inglesi, che quantificano tecnologie complesse, competenze, investimenti, numero di occupati, ricadute su imprese medio-grandi e piccole. Analoghe considerazioni per gli esempi successivi.
} 
mediante analisi, controllo e regolazione a distanza dei flussi, grazie a una serie rilevante di tecnologie disponibili, ma in continua evoluzione. 2) Intraprendere i primi passi verso il passaggio cruciale dalla "produzione e distribuzione centralizzata" di energia alla "produzione e distribuzione decentralizzata", con lo sviluppo di reti e fonti molteplici di generazione energetica; il primo passo in questa direzione è lo sviluppo della smart grid, ovvero la diffusione sistematica di tecnologie e competenze per un uso intelligente dei dispositivi per il controllo e l'uso razionale in qualsiasi luogo.

Sono primi esempi che non escludono, anzi richiedono, altre linee di azione sui comparti produttivi tradizionali. $E^{\prime}$ però evidente che la loro realizzazione dipende in misura fondamentgale da un cambiamento della logica e dell'architettura organizzativa dei processi innovativi. Va da sé che uno degli elementi cruciali è il mutamento di cultura tecnica e manageriale che tutto ciò esige.

Livello attinente alla forma organizzativa delle attività economiche.

Riprendiamo I'aporia fondamentale tra interessi di breve e di lungo termine, in altri termini tra orizzonte immediato di preservazione dell'esistente e scenario di evoluzione del sistema in un lungo arco temporale, entro il quale ci si deve misurare con la transizione socio-tecnica. $E^{\prime}$ in questo ambito concettuale che pensiamo vada ripreso e sviluppato il tema molto importante dei beni comuni (commons), qui riferiti alle risorse essenziali per il mantenimento e la valorizzazione del potenziale di sviluppo a lungo termine di un contesto sistemico.

Nelle economie di mercato i beni comuni culturali, sociali e naturali non ricevono un prezzo e sono sovra o sottoutilizzati dai mercati. Accanto ai settori delle imprese private e pubbliche, emerge l'esigenza di un settore di imprese che gestisca i beni comuni. Il trust potrebbe essere uno strumento giuridico appropriato per la loro gestione strategica e operativa, in quanto consente di separare il godimento dall'amministrazione di un patrimonio. Il disponente trasferisce la proprietà di un bene ad un soggetto fiduciario, che l'amministra per il perseguimento di una certa finalità, affiancato da un guardiano con compiti di consiglio e di vigilanza. La proposta di Peter Barnes (2006) è di creare dei trust (ovvero, all'incirca, delle fondazioni senza scopo di lucro) che diventino proprietarie dei beni comuni, ne concedano un uso razionale ai privati, assegnando loro un valore economico. I beni comuni sarebbero dunque soggetti a un peculiare "regime proprietario" di natura privato-collettiva, senza essere né privatizzati né statalizzati. Quando essi sono scarsi o sotto minaccia, occorrerebbe limitarne I'uso e, grazie ai prezzi richiesti per il loro utilizzo, il trust genererebbe un 
reddito da ripartire tra i cittadini, assicurando un reddito minimo e attenuando la tendenza capitalistica alle disuguaglianze. Quando invece essi sono illimitati, come la cultura o internet, il trust concederebbe, al più basso costo possibile, il maggior beneficio al numero massimo di persone, ulteriormente migliorando l'uguaglianza di ciascuno e il benessere di tutti. In entrambi i casi questo settore dell'economia procederebbe con una logica opposta rispetto a quella del settore privato. Il ruolo dello Stato e delle Istituzioni ad ogni livello risiederebbe -non è poco- nel garantire in ultima istanza i commons; nell'assegnare ai trust i diritti di proprietà; nel finanziare il riacquisto di beni comuni, quali le terre o i diritti sull'acqua, diventati proprietà privata. A nostro parere, la proposta di Barnes si presta con particolare efficacia ad essere sperimentata in una regione, come la Toscana, in cui i commons hanno un rilievo economico ancora più elevato di quello, comunque grande, che rivestono in altri territori. Un'indicazione generale di policy che appare fondato avanzare è pertanto la seguente: soggetti privati e pubblici si impegnino nella creazione di una rete di trust toscani, dedicati ai nostri maggiori commons., per assicurare ad essi strategie di valorizzazione incentrate su visioni di lungo periodo esplicitamente enunciate e sottoposte al pubblico dibattito.

In termini finanziari, i trust dovrebbero nascere da partenariati pubblico/privato in cui anche il capitale privato assuma finalità di pubblico interesse. Appropriati organismi di investimento collettivo possono realizzare tale obiettivo e, senza alcuna pretesa prescrittiva, un esempio può essere tratto dal mondo della finanza etica. Fefisol, uno tra i più noti Fondi europei di finanza solidale, ha la forma giuridica della Sicav (Società d'investimento a capitale variabile), ossia società per azioni avente per oggetto esclusivo I'investimento del patrimonio raccolto mediante l'offerta al pubblico di proprie azioni. Le risorse del Fondo debbono essere collettivamente e obbligatoriamente impiegate in modi stabiliti dallo statuto della società. Inoltre la Sicav ha una forma partecipativa, poiché l'investitore, come azionista, è anche socio e ha diritto di voto nelle assemblee.

\section{Livello concernente lo start-up di imprese innovative}

Poniamoci infine sul terzo ambito strategico-operativo, ovvero quello concernente la creazione e il rafforzamento di impulsi propulsivi verso la trasformazione sociotecnica. Intendiamo riferirci alla tematica delle start-up innovative, al centro di una vasta letteratura teorica ed empirica. Uno dei nodi critici di affrontare in proposito è indubbiamente costituito dalla necessità che le PMI facciano un maggiore ricorso al 
capitale di rischio rispetto alla leva del debito. A questo riguardo il recente Fondo Italiano d'Investimento, promosso dalle maggiori banche, da Confindustria e dal Ministero del Tesoro, può diventare uno strumento cruciale, favorendo la patrimonalizzazione delle imprese con fatturato tra 10 e 100 milioni di euro, oppure la loro aggregazione per un progetto di sviluppo. Su scala regionale, rimane tuttavia un ampio spazio per un Fondo comune d'investimento mobiliare chiuso riservato ad investitori qualificati e rivolto alla microimprenditorialità e allo start-up d'impresa. L'ispirazione, oltreché dall'esperienza di Fidi Toscana, può venire dal Regno Unito, in cui sono stati attivati nove Regional Venture Capital Funds, che sostengono le PMI con alto potenziale di crescita, ma individuandole con criteri diversi dall'una all'altra regione.

Per la prospettiva interpretativa delineata in questo paper, un altro strumento decisivo è il contratto di rete, introdotto in Italia con la legge 33/2009. Esso consiste in un contratto tra imprese che intendono sviluppare insieme un nuovo prodotto 0 servizio, mantenendo ognuna la propria identità e singolarità, ma condividendo un progetto di sviluppo comune. In una logica contrattuale anziché societaria, esso definisce regole dispositive, ossia derogabili dalle parti, e può agire anche attraverso la costituzione di un fondo amministrato da un organo comune e con poteri di stipulare contratti con terzi. Si tratta di un modello più agile del consorzio, avendo una forte struttura pattizia ed una finalità d'innovazione, e in grado di realizzare aggregazioni proprietarie in maniera complementare rispetto alle fusioni e acquisizioni. Esso soprattutto permetterebbe a filiere lunghe e prive di prossimità geografica di realizzare forme di coordinamento migliori di quelle basate su contratti bilaterali (Cafaggi, 2009).

Il contratto di rete appare uno strumento molto interessante proprio nella fase odierna, cioè quando assume particolare rilevanza lo sviluppo di varietà correlate (par. 2.3), viste in termini di network tecnico-produttivi che esplorano spazi di conoscenza in continua evoluzione (transizione socio-tecnica).

Tuttavia, la maggiore debolezza dell'attuale legge è che nel contratto di rete non possono entrare soggetti pubblici e soggetti privati diversi da imprese, quali centri di ricerca o associazioni che potrebbero invece essere essenziali nell'orizzonte appena delineato. A questo proposito si aprono dunque spazi rilevanti per iniziative di livello regionale.

Sembra razionale, infine, ipotizzare un'istituzione regionale di secondo livello (una Fondazione) che selezioni progetti (una o due iniziative all'anno) e vi partecipi con 
investimenti cospicui. I progetti debbono riguardare nuove compagini imprenditoriali, il rinnovamento e la costruzione di nuove linee industriali, connesse a direttrici produttive strategicamente definite (vedi la Terza parte) ${ }^{33}$.

\subsection{SPUNTI DI RIFLESSIONE: SINTESI FINALE}

Il lungo percorso di riflessione sviluppato in questo contributo è iniziato con una rappresentazione di macro-traiettorie evolutive e dei fattori di mutamento di fondamentali proprietà dei SSL. Il passaggio successivo è stata l'individuazione di sfide che si ergono di fronte a sistemi che intendano misurarsi con spazi tecnicoproduttivi soggetti ad un'incessante evoluzione, partendo da interrogativi di fondo sulle proprietà sistemiche essenziali nella fase odierna di transizione socio-tecnica. In relazione a scenari di questa natura sono stati ipotizzati meccanismi strategicooperativi per l'innesco di processi innovativi multi-dimensionali: 1) elaborazione di visioni relative agli scenari che si aprono e si dispiegano, 2) introduzione di dispositivi per favorire la virata di imprese e settori verso nuovi spazi cognitivo-produttivi, 3) creazione di nuove entità in grado di agire da elementi propulsivi.

33 Su queste basi possono essere previsti meccanismi operativi funzionali. Ad esempio sembrano interessanti alcune idee avanzate in Chiocca et al. (2009), cioè che i finanziamenti siano erogati senza interessi per 10-15 anni, purché sia verificabile il mantenimento nel tempo dei requisiti di base (innovazione continua e modelli di coesione sociale elevati) e, dopo i primi cinque anni, si capitalizzino i dividendi eventualmente distribuiti dalle imprese beneficiarie, che vengono reinvestiti nell'istituzione per svilupparla ulteriormente. 


\section{Riferimenti bibliografici}

1. Amin A., Cohendet P., (2003), Architectures of knowledge: firms, capabilities and communities, Oxford University Press, Oxford.

2. Arthur W. B., (1994), Increasing Returns and Path Dependence in the Economy, The University of Michigan Press, Ann Arbor.

3. Bagnasco A., (2003), Società fuori squadra, Il Mulino, Bologna.

4. Baldwin R., (2006), Globalization: the great unbundling(s), Economic Council of Finland, Helsinki.

5. Barabasi A-L., Albert R., (1999), Emergence of Scaling in Random Networks, Science, vol. 286, 15 October, 509-512.

6. Barnes P., (2006), Capitalismo 3.0, EGEA, Milano, 2007.

7. Becattini G., Rullani E., (1993), "Sistema locale e mercato globale", ora in Becattini G., Il distretto industriale, Rosenberg \& Sellier, Torino, 2000

8. Becattini G,. (1991), Il distretto industriale marshalliano come concetto socioeconomico, in Pyke F.- Becattini G. - Sengenberger W. (a cura di), Distretti industriali e cooperazione fra imprese in Italia, Quaderno $\mathrm{n}^{\circ} 34 \mathrm{di}$ Studi e Informazioni, Banca Toscana.

9. Bellandi M., (1989), Capacità innovativa diffusa e sistemi locali di imprese, in Becattini G. (ed.), Modelli locali di sviluppo, Bologna, Il Mulino, pp. 149-173.

10. Bellandi M., (2001), "Local development and embedded large firms", Entreprenuership and Regional Development, vol. 13, n. 3, 189-210.

11. Bell D., (1999), The Coming of Post-Industrial Society, New York, Basic Books.

12. Bertini G., (2007), "L'apprendimento autogestito", Sviluppo \& Organizzazione, n. 223, settembre/ottobre.

13. Boschma R. A., (2005), "Proximity and innovation. A critical assessment", Regional studies, 39(1), 61-74.

14. Boschma R. A., -Iammarino S., (2009), "Related variety, trade linkages and regional growth in Italy", Economic geography, 85(3).

15. Cafaggi F. (2009), a cura di, Il contratto di rete. Commentario, Il Mulino, Bologna.

16. Carley K.M., (2000), Organizational Change and the Digital Economy: A Computational Organization Science Perspective, in: Bryjolfsson E., Kahin B., (eds), Understanding the Digital Economy: Data, Tools, Research, MIT Press, 325-351.

17. Castells M., (2002), The Rise of the Network Society, Oxford, Malden, 2000, trad. it. La nascita della società in rete, Milano, Università Bocconi Editore.

18. Chiocca M., Gardenghi P., Mazzoni L., (2009), "Imprese", in A. Bosi - M. Deriu - V. Pellegrino (a cura di), Il dolce avvenire, Esercizi di immaginazione radicale del presente, Diabasis, Reggio Emilia.

19. Coe N.M.., Hess M., Yeung H.W., (2004) 'Globalizing' regional development: a global production networks perspective, Trans Inst Br Geogr, 29, 468-484.

20. Constant II E.W., (1987), The Social Locus of Technological Practice: Community, System, or Organization?, In: Bijker W.E., Hughes T.P., Pinch T.(Eds.), The Social Construction of Technological Systems: New Directions in the Sociology and History of Technology, MIT Press.

21. DBR (Deutsche Bank Research), (2007), German Mechanical Engineering. Opportunities beckon in global growth centres, July, 10.

22. DBR (Deutsche Bank Research), (2008), German Mechanical Engineering steeling economy for the post-oil era, December, 16.

23. DBR (Deutsche Bank Research), (2009a), Crisis year 2009 accelerating pace of structural change in Germany, August, 20.

24. DBR (Deutsche Bank Research), (2009b), German electrical and electronic industry, August, 18.

25. Dei Ottati, G. (2009), "Semi-automatic and deliberate actions in the evolution of industrial districts", in G. Becattini et al. (eds.), A handbook of industrial districts, Edward Elgar, Cheltenham, pp.204-215. 
26. Ernst D., Kim L., 2002, Global Production Networks, knowledge diffusion, and local capability formation, Research policy, 31: 1417-1429.

27. Fleming L., Sorenson O., (2001), Technology as a complex adaptive system: evidence from patent data, Research Policy, 30, 1019-1039.

28. Fleming L., Sorenson O., (2004a),Science as a map in technological search, Strategic Management Journal, 25, 909-928.

29. Fleming L., Sorenson O., (2004b), Science and the diffusion of knowledge, Research Policy, 33, 1615-1634.

30. Foxon T., (2006), Bounded rationality and hierarchical complexity: Two paths from Simon to ecological and evolutionary economics, Ecological Complexity, 3, 361-368.

31. Freeman C., Soete L., (1994), Lavoro per tutti o disoccupazione di massa?, Etaslibri Milano.

32. Frenken, K. - van Oort, F. G. - Verburg, T. (2007), "Related variety, unrelated variety and regional economic growth", Regional studies, 41(5), pp.685-697.

33. Geels F. W. (2002), Technological transitions as evolutionary reconfiguration processes: a multi-level perspective and a case-study, Research Policy, 31,1257-1274.

34. Geels, F. W. (2004), "From sectoral system of innovation to socio-technical systems", Research policy, 33, pp.897-920.

35. Geels, F. W. (2005), Processes and patterns in transitions and system innovations: Refining the co-evolutionary multi-level perspective, Technological Forecasting \& Social Change, 72, 681-696.

36. Geels, F. W., Kemp R., (2007), Dynamics in socio-technical systems: Typology of change processes and contrasting case studies, Technology in Society, 29, 441-455.

37. Gereffi G., (2001), Shifting Governance Structures in Global Commodity Chains, With Special Reference to the Internet, American Behavioral Scientist, 1616-1637.

38. Gereffi G., Humphrey J. Sturgeon T., (2005), The governance of global value chains, Review of International Political Economy, 12:1 February, 78-104.

39. Gilson R. J., Sabel C. F., Scott R. E., (2009), "Contracting for innovation: vertical disintegration and interfirm collaboration", Columbia law review, 109(3), 431-502.

40. Granovetter M.S, (19739, The Strength of Weak Ties, American Journal of Sociology, Vol. 78, Issue 6, May, 1360-1380.

41. Grossman G., Rossi-Hansberg E., (2006), "The rise of offshoring: it's not wine for cloth anymore", paper presented at Kansas Fed's Jackson Hole conference for Central Bankers, http://www.kc.frb.org/

42. Guidi F. (2009), "Filiera corta: percorsi di innovazione tecnici, organizzativi e sociali nella gestione strategica delle nicchie", Tesi di Dottorato in Cooperazione internazionale e politiche per lo sviluppo sostenibile, Università di Bologna.

43. Hamdouch A., (2009), "Networking, clustering and innovation dynamics in the global economy: general presentation", Journal of innovation economics, 2, pp.5-13.

44. Hamdouch A., Depret M.-H., (2009), "Surveying the literature on territorial innovation systems: a suggested interpretation framework", SSRN working paper.

45. Hausmann R., Klinger B., Wagner R., (2008), "Doing growth diagnostics in practice: a 'mindbook" , Harvard University Center for International Development, Working Paper n.177.

46. Hekkert M.P., Negro S. O., (2009), Functions of innovation systems as a framework to understand sustainable technological change: Empirical evidence for earlier claims, Technological Forecasting \& Social Change, 76, 584-594.

47. Hidalgo C. A., Klinger B., Barabási A.-L., Hausmann R., (2007), "The product space conditions the development of nations", Science, 317, 482-487.

48. Hidalgo C. A., (2009), "The dynamics of economic complexity and the product space over a 42 years period", CID working paper, n. 189.

49. Hughes T.P., (1987), The evolution of large technological systems. In: Bijker W.E., Hughes T.P., Pinch T.(Eds.), The Social Construction of Technological Systems: New Directions in the Sociology and History of Technology, MIT Press.

50. IRPET (2009), Il mercato del lavoro. Rapporto 2008, Regione Toscana, Firenze. 
51. Katz J. S., (2006), Indicators for complex innovation systems, Research Policy, 35, 893-909.

52. Kemp R., Schot J., Hoogma R., (1998), Regime Shifts to Sustainability Through Processes of Niche Formation: The Approach of Strategic Niche Management, Technology Analysis \& Strategic Management, Vol. 10, No. 2, 175-195

53. Krugman P., (1991), Geoghaphy and trade, MIT Press, Cambridge.

54. La Spina A., (2003), La politica per il Mezzogiorno, Il Mulino, Bologna.

55. Lombardi M., (2006), Forme di cooperazione tra unità economiche: basi cognitive ed evoluzionistiche, in G. Cainelli e N. De Liso (a cura di), Dinamica dei sistemi di imprese, in corso di stampa pesso Franco Angeli,

56. Lombardi M., (2008), A morphogenetic approach to the evolution of technological capabilities, in R. Leoncini e A. Montresor (eds), Dynamic Capabilities Between Firm Organisation and Local Systems of Production, Routledge,ctions in the Sociology and History of Technology, MITP ress, Cambridge, MA, 51-82.

57. Lombardi M., Salani P., Baroni M., (2009), "The evolutionary trajectories of countries within the product space of international trade", Oxford journal of business and economics, December, 31-47.

58. Lombardi M., (2010)The production process as a complex, dynamic and ordered world, in: De Liso N. Leoncini R. (Eds), Internationalization, Technological Change and the Theory of the Firm, Routldge.

59. Lombardi M., Macchi M., (2010), Strategie di aiuto allo sviluppo: windows of opportunity e bootstrapping strategies, in M. Biggeri, L. Canitano (eds, in corso di stampa).

60. Markard J. Truffer B., (2008), Actor-oriented analysis of innovation systems: exploring micro-meso level linkages in the case of stationary fuel cell, Technology Analysis \& Strategic Management, Vol. 20, No.4, July,443-464.

61. Marshall A. (1890), Principles of economics, Macmillan, London.

62. Mokyr J., (1990) "Punctuated Equilibria, and Technological Progress", American Economic Review, May, 350-354. Mokyr J.(2002), "Innovation in an Historical perspective: Tales of Technology and Evolution", in Steil B., Victor D.G., Nelson R.R., (eds), Technological Innovation and Economic Performance, Princeton University Press, Princeton.

63. Mokyr J., (2002), "Innovation in an Historical perspective: Tales of Technology and Evolution", in Steil B., Victor D.G., Nelson R.R., (eds), Technological Innovation and Economic Performance, Princeton University Press, Princeton.

64. March J. G., (1991), Exporation and Exploitation in Organizational Learning, Organization Science, 2, 1, 71-87.

65. March J. G., Simon H., (1958-1993), Organization, Backwell, Cambridge MASS.

66. Markar J., Truffard B., (2008), Technological innovation systems and the multi-level perspective: Towards an integrated framework, Research Policy, 37, 596-615.

67. Morrison M.; Pietrobelli C.; Rabellotti R., (2008), Global Value Chains and Technological Capabilities: A Framework to Study Learning and Innovation in Developing Countries, Oxford Development Studies, Vol. 36, N.1, 39-58.

68. Newman M.E.J., (2001), The structure of scientific collaboration networks, Proceedings of National Academy of Science PNAS, 88, 2, 404-409.

69. Newman M.E.J., (2004), Coauthorship networks and patterns of scientific collaboration, Proceedings of National Academy of Science PNAS, 101, 1, 5200-5205..

70. Nooteboom, B. (2004), Inter-firm collaboration, learning and networks: an integrated approach, Routledge, London.

71. OECD, (2007), Moving Up the Value Chain: Staying Competitive in the Global Economy

72. Ostrom E., Janssen M.A., Anderies J.A, (2007), Going beyond panaceas, Proceedings of National Academe of Science PNAS, vol. 104, 39, 15176-15178.

73. Ozman M., (2009), Interfirm networks and innovation: a survey of literature, Economic of Innovation and New Technology, Vol.18, No.1, January,39-67.

74. Paavola S., Hakkarainen K., (2005), "The knowledge creation metaphor: an emergent epistemological approach to learning", Science \& Education, 14(6), pp.535-557. 
75. Perez C., (2001), Technological change and opportunities for development as a moving target, Cepal Review 75, December, 109-130.

76. Perez C., (2004), Technological revolutions, paradigm shifts and socio-institutional change, Reinert, Erik (ed) Globalization, Economic Development and Inequality: An alternative Perspective, Edward Elgar, Cheltenham, UK; Northampton, MA, USA, 2004, 217-242

77. Porter M. E., (1990), The competitive advantage of nations, The Free Press, New York.

78. Rammel C., van den Berg J., (2003), Evolutionary policies for sustainable development: adaptive flexibility and risk minimizing, EcologicalEconomics, 47, 121-133.

79. Rip. A., Kemp M., (1998), "Technological change", in Rayner, S. - Malone, E. L. (Eds.), Human choice and climate change, vol.2, Batelle Press, Washington DC, pp.327-399.

80. Rip A., Kemp R., 1998,Technological change, In: Rayner S., Malone E.L.(Eds), Human Choice and Climate Change, Vol. 2, Battelle Press, Columbus, OH, 327-399.

81. Roberts, J,. (2004), L'impresa moderna, Il Mulino, Bologna, 2006.

82. Rocky Mountains Institute (2004), Winning the Oil Endgame, http://www.rmi.org/rmi/

83. Rodrik D., (2008), "Second best institutions", American economic review, may.

84. Rosenbloom R. S., Spencer W. J. (eds.), 1996, Engines of innovation: U.S. industrial research and the end of an era, Harvard Business School Press, Boston.

85. Rouse W. B., Baba M.L., (2006), Enterprise Transformation, COMMUNICATIONS OF THE ACM July 2006/Vol. 49, No. 7: 67-72.

86. Rycroft R., Kash D., (2002), Path Dependence in the Innovation of Complex Technologies, Technology Analysis \& Strategic Management, Vol. 14, No.1

87. Sachs J. D., (2005), La fine della povertà, Mondadori, Milano.

88. Scarlato M., (2010), "Lo sviluppo del Mezzogiorno: come superare lo stallo?", Working paper n.112, Dipartimento di Economia, Università di RomaTre.

89. Shibata N., Kajikawa Y., Takeda Y., Matsushima K., (2008), Detecting emerging research fronts based on topological measures in citation networks of scientific publications, Technovation,2, 758-775.

90. Storper M., (2009), "Regional context and global trade", Economic geography, January.

91. UNCTAD, 2007, The Least Developed Countries Report, 2007: Knowledge, technological learning and innovation for development,

92. UNIDO, Unite Nations of Industrial Development Organization, (2005), Industrial Development Report 2005. Capability building for catching-up Historical, empirical and policy dimensions, Vienna.

93. Unruh G. C., (2002), Escaping carbon lock-in, Energy Policy, 30, 317-325.

94. Unruh G. C., Hermosilla J.C., (2006), Globalizing carbon lock-in, Energy Policy, 34, 1185-1197.

95. Van de Poel I., (2003), The transformation of technological regime, Research Policy, 49-68.

96. Viesti G., (2000), Come nascono i distretti industriali, Laterza, Bari-Roma.

97. Van Raan A.F.J., van Leeuwen Th.N., (2002), Assessment of the scientific basis of interdisciplinary, applied research. Application of bibliometric methods in Nutrition and Food Research, Research Policy, 31, 611-632.

98. Verbong G., .Geel F.W., Raven R., (2008), Multi-niche analysis of dynamics and policies in Dutch renewable energy innovation journeys (1970-2006): hyper-cycles, closed networks and technology-focused learning, Technology Analysis \& Strategic Management, Vol.20, No.5, ,555-573

99. Wagner C.W., Leydesdorff L., (2005), Network structure, self-organization, and the growth of international collaboration in science, Research Policy , 34, 1608-1618.

100. Wagner C.W., Leydesdorff L., (2006), Measuring the globalization of knowledge networks, Paper presented at "Blue Sky II 2006:" What Indicators for Science, Technology and Innovation Policies in the 21st Century. Paris: OECD; at http://www.oecd.org/document.

101.Wagner C.W., Leydesdorff L., (2008), International collaboration in science and the formation of a core group, Journal of Informetrics, 2, 3,17-325. 
102. Watts D. J., (1999). Small Worlds: The Dynamics of Networks Between Order and Randomness. Princeton University Press.

103. Weitzman M. L., (1996), Hybridizing Growth Theory, American Economic Theory, vol. 86, 2, 207-212.

104. Weitzman M. L., (1998), Recombinant Growth, The quarterly Journal of Economics, vol CXIII, 2, 331-360.

105. Yi K-M., (2003), Can Vertical Specialization Explain the Growth of World Trade?, Journal of Political Economy, University of Chicago Press, vol. 111(1), 52-102, February.

106. Zysman J., (2006), The $4^{\text {th }}$ Service Transformation: The Algorithmic Revolution, CACM Special Issue on Services Science, July. 